Article

\title{
Oenological Characteristics of Fermented Apple Musts and Volatile Profile of Brandies Obtained from Different Apple Cultivars
}

\author{
Magdalena Januszek*(iD), Paweł Satora $\mathbb{D}$ and Tomasz Tarko $\mathbb{D}$ \\ Department of Fermentation Technology and Microbiology, Faculty of Food Technology, \\ University of Agriculture in Krakow, Balicka Street 122, 30-149 Krakow, Poland; pawel.satora@urk.edu.pl (P.S.); \\ tomasz.tarko@urk.edu.pl (T.T.) \\ * Correspondence: magdalena.kostrz@urk.edu.pl
}

Received: 6 May 2020; Accepted: 31 May 2020; Published: 3 June 2020

check for updates

\begin{abstract}
Volatile profile of spirits is the most important factor, because it can contribute to pleasant flavor. The aim of the study was to determine the impact of dessert apple cultivar used for fermentation on the concentration of volatile compounds in apple spirits. SPME-GC-MS (solid-phase microextraction- gas chromatography- mass spectrometry) method enables the detection of 69 substances and GC-FID (gas chromatography - flame ionization detector) 31 compounds. Characteristic volatiles for brandies obtained from Topaz were limonene, myrcene, methyl valerate and 1,1-diethoxy-propane, from Rubin- $\beta$-citronellol and isopropyl acetate, Elise-limonene, myrcene benzyl acetate and isopropyl acetate, Szampion- $\beta$-citronellol, Idared-1,1-diethoxy-propane and Jonagored-ethyl trans-4-decanoate. Of the ten analyzed apple spirits, those obtained from Topaz, Rubin and Elise cultivars demonstrated the most diverse profile of volatile compounds. Moreover, their oenological parameters that are the most important in the production of alcoholic beverages were the most favorable. On the other hand, the content of sugars was relatively low in Elise must, while it was highest in Topaz must, which later on translated into differences in alcohol content. Brandies obtained from Gloster contained the smallest concentrations of esters and terpenes. Results of the sensory analysis showed that highest rated brandies were obtained from Topaz, Rubin, Elise and Florina.
\end{abstract}

Keywords: apple brandies; volatile compounds; terpenes; esters; sensory analysis

\section{Introduction}

Apple tree (Malus domestica) is most frequently grown fruit tree in the Poland. Most of the harvested apples are processed - they are mainly used for the production of apple juice concentrate (about $90 \%$ ), fresh juices, smoothies, droughts and alcoholic beverages [1]. The quality of alcoholic beverages is closely dependent on the quality of raw materials, yeast applied for fermentation and fermentation conditions. Fruits designated for processing should reach suitable maturity. The aroma of overripe fruits may be characterized by non-specific aroma notes associated with the development of epiphytic microbiota, while unripe fruit contain less sugar and are not suitable for the production of high-quality beverages [2]. Apart from compounds derived from raw material, volatiles are also formed in biologic, enzymatic and chemical processes throughout whole ethanol fermentation. Esters, aldehydes, higher alcohols, organic acids and terpenes are the most important aroma compounds of alcoholic beverages [3,4].

Esters are derived from raw materials, produced by yeast during fermentation in the reaction between alcohols and acetyl-CoA catalyzed by acetyltransferase, while some may be formed by transesterification as well. The process of ester formation is influenced by many factors, including 
fermentation temperature, $\mathrm{pH}$, nitrogen level, microbiota present during fermentation and factors stimulating microbial growth [3].

Terpenes is a large group of aliphatic long-chain hydrocarbons, derived from active isoprene (isopentenyl diphosphate) or its isomer-dimethylallyl pyrophosphate. They are formed by combining two or more 5-carbon-atoms molecules leading to the formation of $\mathrm{C} 10$ monoterpenes, $\mathrm{C} 15$ sesquiterpenes or C20 diterpenes. There are various types of oxygenated derivatives of terpene hydrocarbons including alcohols (citronellol and linalool), aldehydes (citral and citronellal), ketones (mircenon and $o$-cymenon), esters or oxides [3].

Higher alcohols constitute between $0.1 \%$ to $0.7 \%$ of the quantity of produced ethanol. According to the Ehrlich theory, fusel alcohols are generated by decarboxylation and deamination of corresponding amino acids, for example, leucine is converted to 3-methyl-1-butanol, isoleucine to 2-methyl-1-butanol and valine to isobutanol [3,5].

Most data regarding volatile profile and sensory analysis of fruit brandies regards the determination of qualitative and quantitative profiles of volatile compounds, but it does not concern the impact of particular cultivars on such chemical substances in alcoholic beverages [6-8]. It seems that in the case of brandies produced from various cultivars of plums [9], cherries and pears [10] the selection of certain cultivars had the major impact on the profile of volatile compounds, and it was related to highest results obtained in sensory analysis. There are relatively few studies on apple brandies regarding the impact of apple cultivar used for fermentation on detailed profile of volatile compounds of apple distillates. Moreover, dessert apples are increasingly used to produce apple brandies. Therefore, in our experiments we used cultivars of dessert apple most commonly grown not only in Poland, but also in many other countries. The aim of the study was to determine the impact of dessert apple cultivar used for fermentation on the composition and concentration of volatiles in apple spirits. The results of our research will enrich the knowledge about the effect of the fruit cultivar on the quality of brandies-and also enable producers to choose a cultivar of apples to produce beverages with characteristic flavor.

\section{Materials and Methods}

\subsection{Fermentation}

Apple musts used for fermentation were obtained from ten different apple cultivars (Elise, Rubin, Topaz, Golden delicious, Szampion, Gloster, Pinova, Florina, Idared and Jonagored). Fruits were harvested in orchards in Garlica Murowana $\left(50.1500^{\circ} \mathrm{N}, 19.9333^{\circ} \mathrm{E}\right.$, Małopolska district, Poland). Apples were washed, crushed, pressed and divided into $2 \mathrm{~kg}$ aliquots in $3 \mathrm{~L}$ sterile glass flasks. Musts were supplemented with $\left(\mathrm{NH}_{4}\right)_{2} \mathrm{HPO}_{4}(0.2 \mathrm{~g} / \mathrm{kg}$ raw fruit) and inoculated $(0.3 \mathrm{~g}$ dry weight $/ \mathrm{L}$ of must) with Ethanol Red (Saccharomyces cerevisiae) yeast strain (Starowar, Warsaw, Poland). Alcoholic fermentation was carried out for 30 days at $20^{\circ} \mathrm{C}$. Weight loss associated with the liberation of carbon dioxide was measured daily.

\subsection{Distillation}

First, fermented musts were distilled till the ethanol concentration in the collected distillate was lower than $0.5 \%(w / v)$. Then collected distillate was distilled as well and final ethanol concentration was ranging between $11.6-20.2 \%(v / v)$ of ethanol.

Then, the distillate was distilled again using a glass column $(40 \mathrm{~cm})$ filled up to $60 \%$ with Raschig rings and three fractions were collected: the heads ( $2 \%$ of the distillate), the heart fraction $(83 \%)$ and the tails (15\%). Final ethanol concentrations in apple brandies was approximately $65 \%(v / v)$, in head fraction $80 \%(v / v)$ and in tail fraction $20 \%(v / v)$, respectively. In order to avoid the loss of volatiles all fractions were kept at $4{ }^{\circ} \mathrm{C}$ in sealed flasks until further analysis. In the current study, we only presented results for heart fraction. 


\subsection{Analysis of Oenological Parameters}

The ethanol content, total extract and sugar-free extract were determined using officially approved methods [11]. Titratable acidity (TA) was determined using Mettler DL 25 titrator (Greifensee, Switzerland). It was calculated from the volume of $0.1 \mathrm{M} \mathrm{NaOH}$ used for titration and expressed as gram of malic acid per liter. Fermentation efficiency (\%) was calculated based on the relationship between sugar loss and ethanol produced following the fermentation stoichiometry, where $0.511 \mathrm{~g}$ or $0.538 \mathrm{~g}$ ethyl alcohol is obtained from $1 \mathrm{~g}$ of reducing sugars or sucrose, respectively. Free amino nitrogen (FAN) was determined with the ninhydrin method. The absorbance of samples was measured at a wavelength $\lambda=575 \mathrm{~nm}[12]$.

\subsection{Determination of Sugar Content by High Performance Liquid Chromatography}

Samples of apple musts before and after fermentation were centrifuged (MPW-65R, MPW Med. Instruments, Warszawa, Poland) at $14,000 \times \mathrm{g} / 5 \mathrm{~min}$ and fresh musts were diluted. Fermented musts were evaporated (Rotavapor R-220 SE, Buchi AG, Flawil, Switzerland) prior to analysis. Before injecting samples, we filtered them through syringe filters $(0.45 \mu \mathrm{m}$ pore density, Sartorius AG, Getinge, Germany). The analysis of sugar profile was carried out by high performance liquid chromatography (HPLC) method using Shimadzu apparatus (Kyoto, Japan) NEXERA XR equipped with the refractometer detector RF-20A. Separation was performed on the Asahipak NH2P-50, $4.6 \times 250 \mathrm{~mm}$ Shodex column (Showa Denko America, Munich, Germany) thermostated at $30^{\circ} \mathrm{C}$. An aqueous solution of acetonitrile $(70 \%)$ was the mobile phase and isocratic program elution $(0.8 \mathrm{~mL} / \mathrm{min})$ lasted $16 \mathrm{~min}$. Standard curves were prepared for the following substances: glucose, fructose, sucrose and glycerol. To validate the method we measured the concentration of mentioned substances in ten apple musts before and after fermentation and then we added known quantities (5, 10 and $20 \mathrm{~g} / \mathrm{L})$ of sugars or glycerol to those musts and carried out measurements again. We carried out that analysis in three replicated and we confirmed that added quantities were detected. Considering low detection limits (LOD) and low quantity limits (LOQ) (Table S1, Supplementary Materials) we confirmed that applied method was suitable for testing musts before and after fermentation. Moreover, R2 values indicated very high linearity within tested range of used standards.

\subsection{Volatile Compounds Analysis by Gas Chromatography-Flame Ionization Detector and Solid Phase Microextraction-Gas Chromatography-Mass Spectrometry}

Analysis of selected volatile compounds was carried out using gas chromatography as described by Satora and Tuszyński (2005) [13]. Gas chromatography-flame ionization detector (GC-FID) analysis was carried out on the Hewlett Packard 5890 Series II chromatograph system. Tested components were separated on the HP-INNOWAX capillary column (crosslinked polyethylene glycol stationary phase; $30 \mathrm{~m} \times 0.53 \mathrm{~mm}$ ID with $1.0 \mu \mathrm{m}$ film thickness, Agilent, Santa Clara, CA, USA). the temperature of detector and injector was $250^{\circ} \mathrm{C}$ and the column was heated using the following temperature program: $35{ }^{\circ} \mathrm{C}$ for five minutes at increments of $5^{\circ} \mathrm{C} / \mathrm{min}$ to $110{ }^{\circ} \mathrm{C}$, then $40{ }^{\circ} \mathrm{C} / \mathrm{min}$ to $220^{\circ} \mathrm{C}$ and maintained at constant temperature for three minutes. The carrier gas was helium at a $20.0 \mathrm{~mL} / \mathrm{min}$ flow. Hydrogen flow speed was $33.0 \mathrm{~mL} / \mathrm{min}$, and that of air was $400 \mathrm{~mL} / \mathrm{min}$. Qualitative and quantitative identification of volatile substances (Sigma-Aldrich, Saint Louis, MO, USA) was based on the comparison of retention times and peak surface area read from sample and standard chromatograms and verified against results obtained for the internal standards (anethole, ethyl nonanoate and 4-methylo-2-pentanol). Concentrations of volatile components were recalculated based on $100 \%(v / v)$ ethanol and were expressed as $\mathrm{mg} / \mathrm{L}$.

In solid phase microextraction-gas chromatography-mass spectrometry (SPME-GC-MS) method, $2 \mathrm{~mL}$ of saturated saline with an internal standard solution (5 mg/L 4-methyl-2-pentanol and 0.05 $\mathrm{mg} / \mathrm{L}$ ethyl nonanoate, Sigma-Aldrich) and $0.05 \mathrm{~mL}$ of spirit was added into $10 \mathrm{~mL}$ vials. The SPME device (Supelco, Inc., Bellefonte, PA, USA) coated with PDMS (polydimethylsiloxane), $100 \mu \mathrm{m}$ fiber was first conditioned by inserting it into the GC injector port at $250{ }^{\circ} \mathrm{C}$ for $1 \mathrm{~h}$. For sampling, the fiber 
was inserted into the headspace under stirring (300 rpm) for $30 \mathrm{~min}$ at $60{ }^{\circ} \mathrm{C}$. Subsequently, the SPME device was introduced into the injector port of the Agilent Technologies 7890B chromatograph system equipped with LECO Pegasus HT, high throughput TOF-MS (time-of-flight mass spectrometry), and was kept in the inlet for $3 \mathrm{~min}$. The SPME process was automated using the GERSTEL MultiPurpose Sampler (MPS, GERSTEL Inc., Linthicum, WA, USA).

Analyzed compounds were separated on a Rtx-1ms capillary column (Crossbond 100\% dimethyl polysiloxane, $30 \mathrm{~m} \times 0.53 \mathrm{~mm} \times 0.5 \mu \mathrm{m})$. The detector temperature was $250{ }^{\circ} \mathrm{C}$, and the column was heated using the following temperature program: $40{ }^{\circ} \mathrm{C}$ for three minutes at an increment of $8{ }^{\circ} \mathrm{C} / \mathrm{min}$ to $230^{\circ} \mathrm{C}$, then maintained at constant temperature for $9 \mathrm{~min}$. Carrier: helium at $1.0 \mathrm{~mL} / \mathrm{min}$ constant flow. Electron impact mass spectrometry (EIMS) electron energy $70 \mathrm{eV}$; ion source temperature and connection parts: $250{ }^{\circ} \mathrm{C}$. Analyte transfer was performed in splitless mode; the mass spectrometer-detector (MSD) was set to scan mode from $m / z=40$ to $m / z=400$.

Compounds were identified using mass spectral libraries and linear retention indices, calculated from a series of $n$-alkanes from $C_{6}$ to $C_{30}$. The quantity of volatiles was determined semi-quantitatively by measuring the relative peak area of each identified compound, according to the NIST (National Institute of Standards and Technology) database, in relation to that of the internal standard.

\subsection{Sensory Analysis}

Sensory analysis of apple brandies was based on aroma and included eight sensory descriptors (fruity, sweet, grassy, floral, smoked, citrus, pungent, yeast) rated in 5-point hedonistic scale in quantitative descriptive analysis (QDA). Panelists were selected among scientific staff working in the faculty of food technology and human nutrition who previously graduated from that faculty and obtained extensive course of sensory analysis as a part of their curriculum. Aroma evaluation was determined using a set of standards provided to panelists prior to analysis [14]. First, panelists received standards of various aromas determine whether they were able to recognize each of them. Then they received the same standards, but at various concentrations. Only those who passed those two stages were selected as panelists. Apple brandies (diluted to $40 \%$ vol. EtOH) were subjected to sensory assessment by the panel comprising of 10 panelists. Samples were coded and provided to panelists in randomized order. Results were subjected to one-way analysis of variance (ANOVA) an than Pearson test was carried out for each descriptor (Table S2, Supplementary Materials).

\subsection{Statistical Analysis}

All experiments were performed at least in five replicates and results were presented as arithmetic means \pm standard deviation. Statistical analysis was carried out in the R 3.5 .0 (Vienna, Austria) program. The ANOVA was carried out using linear model (lm) function and Tukey's test was done using honest significant difference (HSD) test function in agricolae'package.

\section{Results and Discussion}

\subsection{Selected Chemical Parameters of Fresh and Fermented Apple Musts}

Fresh, matured apples contain about 10-13\% total sugars, among which fructose dominates [15]. Fructose was dominant in sugar profile (over 50\% of total sugar) in analyzed apple musts (Table 1). The average concentration of glucose was much lower $(9.7-25.5 \mathrm{~g} / \mathrm{L})$. There are various organic acids present in apple fruit, including malic, citric, succinic, quinic and galacturonic acids. These acids occur as free molecules or they are bound to other compounds in must. Their concentration has a significant impact on taste, $\mathrm{pH}$, fermentation and beverage stability [16]. The acidity of certain musts used in the experiment were slightly lower than those found in the studies by Tarko et al. (2018) [17], nonetheless, the acidity increased after fermentation and ranged from 3.73 to $7.32 \mathrm{~g} / \mathrm{L}$. According to legal regulations in Poland [18], total acidity in fermented fruit beverages should range from $3.5 \mathrm{~g}$ to $7 \mathrm{~g}$ of malic acid per liter. 
Table 1. Sugar composition of fresh and fermented musts obtained from various apple cultivars.

\begin{tabular}{|c|c|c|c|c|c|c|c|c|}
\hline \multirow{3}{*}{$\begin{array}{l}\text { Apple } \\
\text { Cultivars }\end{array}$} & \multicolumn{4}{|c|}{ Before Fermentation Processes } & \multicolumn{4}{|c|}{ After Fermentation Processes } \\
\hline & Glycerol & Fructose & Glucose & Sucrose & Glycerol & Fructose & Glucose & Sucrose \\
\hline & \multicolumn{8}{|c|}{$(\mathrm{g} / \mathrm{L})$} \\
\hline Elise & $0.00 \pm 0.00$ & $56.41 \mathrm{e} \pm 1.81$ & $9.71 \mathrm{e} \pm 0.71$ & $14.71 \mathrm{~d} \pm 0.62$ & $4.74 \mathrm{ab} \pm 0.24$ & $0.53 a b \pm 0.36$ & $0.25 \mathrm{abc} \pm 0.02$ & $0.16 \mathrm{a} \pm 0.13$ \\
\hline Rubin & $0.00 \pm 0.00$ & $61.12 \mathrm{a} \pm 0.93$ & $25.52 \mathrm{a} \pm 0.33$ & $13.42 \mathrm{~cd} \pm 0.44$ & $5.57 \mathrm{ab} \pm 1.06$ & $0.52 \mathrm{ab} \pm 0.19$ & $0.08 b c \pm 0.04$ & $0.08 \mathrm{a} \pm 0.03$ \\
\hline Topaz & $0.00 \pm 0.00$ & $70.31 c \pm 0.81$ & $24.94 b \pm 0.61$ & $10.52 d \pm 1.11$ & $5.77 \mathrm{ab} \pm 0.72$ & $0.25 \mathrm{ab} \pm 0.13$ & $0.12 \mathrm{abc} \pm 0.06$ & $0.13 a \pm 0.04$ \\
\hline Szampion & $0.00 \pm 0.00$ & $65.41 c \pm 0.21$ & $14.71 \mathrm{~d} \pm 0.92$ & $17.62 b \pm 0.53$ & $4.94 \mathrm{ab} \pm 0.38$ & $0.86 a \pm 0.24$ & $0.20 \mathrm{ab} \pm 0.02$ & $0.12 \mathrm{a} \pm 0.11$ \\
\hline $\begin{array}{l}\text { Golden } \\
\text { delicious }\end{array}$ & $0.00 \pm 0.00$ & $47.61 b c \pm 0.11$ & $17.43 c \pm 0.83$ & $24.31 \mathrm{a} \pm 1.82$ & $7.26 \mathrm{a} \pm 1.95$ & $0.31 \mathrm{ab} \pm 0.25$ & $0.21 \mathrm{a} \pm 0.13$ & $0.25 \mathrm{a} \pm 0.17$ \\
\hline Jonagored & $0.00 \pm 0.00$ & $54.73 d \pm 0.91$ & $20.42 b \pm 0.61$ & $15.91 b c \pm 0.72$ & $3.88 b \pm 0.39$ & $0.42 \mathrm{ab} \pm 0.11$ & $0.03 c \pm 0.05$ & $0.11 \mathrm{a} \pm 0.08$ \\
\hline Pinova & $0.00 \pm 0.00$ & $56.42 c \pm 0.51$ & $21.93 b \pm 1.32$ & $18.81 b \pm 0.75$ & $4.28 \mathrm{ab} \pm 0.91$ & $0.28 \mathrm{ab} \pm 0.17$ & $0.28 a b c \pm 0.16$ & $0.10 \mathrm{a} \pm 0.07$ \\
\hline Idared & $0.00 \pm 0.00$ & $60.31 \mathrm{ab} \pm 0.01$ & $15.61 \mathrm{~cd} \pm 0.91$ & $23.21 a \pm 0.02$ & $6.27 a b \pm 1.26$ & $0.17 \mathrm{~b} \pm 0.19$ & $0.04 c \pm 0.11$ & $0.03 a \pm 0.03$ \\
\hline Florina & $0.00 \pm 0.00$ & $51.92 d \pm 1.61$ & $14.64 \mathrm{~d} \pm 1.42$ & $17.21 b \pm 2.71$ & $4.32 \mathrm{ab} \pm 1.73$ & $0.67 a b \pm 0.25$ & $0.03 c \pm 0.04$ & $0.06 a \pm 0.03$ \\
\hline Gloster & $0.00 \pm 0.00$ & $50.01 \mathrm{f} \pm 0.41$ & $16.91 f \pm 0.21$ & $16.11 b c \pm 1.41$ & $4.59 \mathrm{ab} \pm 1.11$ & $0.25 a b \pm 0.13$ & $0.03 c \pm 0.04$ & $0.06 a \pm 0.03$ \\
\hline Significance & ns & $* * *$ & $* * *$ & $* * *$ & * & * & ** & ns \\
\hline
\end{tabular}

Same letters next to mean values within columns indicate the lack of statistically significant differences at $p<0.05 ; n=3 ; n s-$ not significant; $0.001={ }^{* * *} ; 0.01={ }^{* *} ; 0.05={ }^{*}$. 
The nitrogen fraction of apples includes amino acids such as asparagine, glutamine, aspartic acid, glutamic acid and serine, which dominate the profile of amino acids ( $86 \%$ to $95 \%$ of total amino acids) and can be easily assimilated by yeast. The content of nitrogen in apples depends on the age of orchards, area of cultivation and the type and amount of used fertilizers. Fruits harvested from trees growing on intensely fertilized soils can contain up to five times higher concentrations of nitrogen compounds than on average [19]. Fresh musts analyzed in our studies contained relatively low amounts of nitrogen compounds (13.7-61.7 mg/L), therefore, the supplementation with ammonium hydrogen phosphate was necessary.

Musts obtained from different apple cultivars demonstrated variable fermentation dynamics (Figure 1). The turbulent fermentation phase began first, in Gloster and Florina musts, while in other samples it was observed about a day later. Similar phenomenon was observed by Satora et al. (2008) [20], who stated that musts obtained from Gloster cultivar fermented earlier than musts obtained from other cultivars. The optimal fermentation rate was noted for Golden delicious musts- the turbulent fermentation lasted about eight days and highest final weight losses were recorded (about $5 \mathrm{~g} / 100 \mathrm{~mL}$ ) (Figure 1).

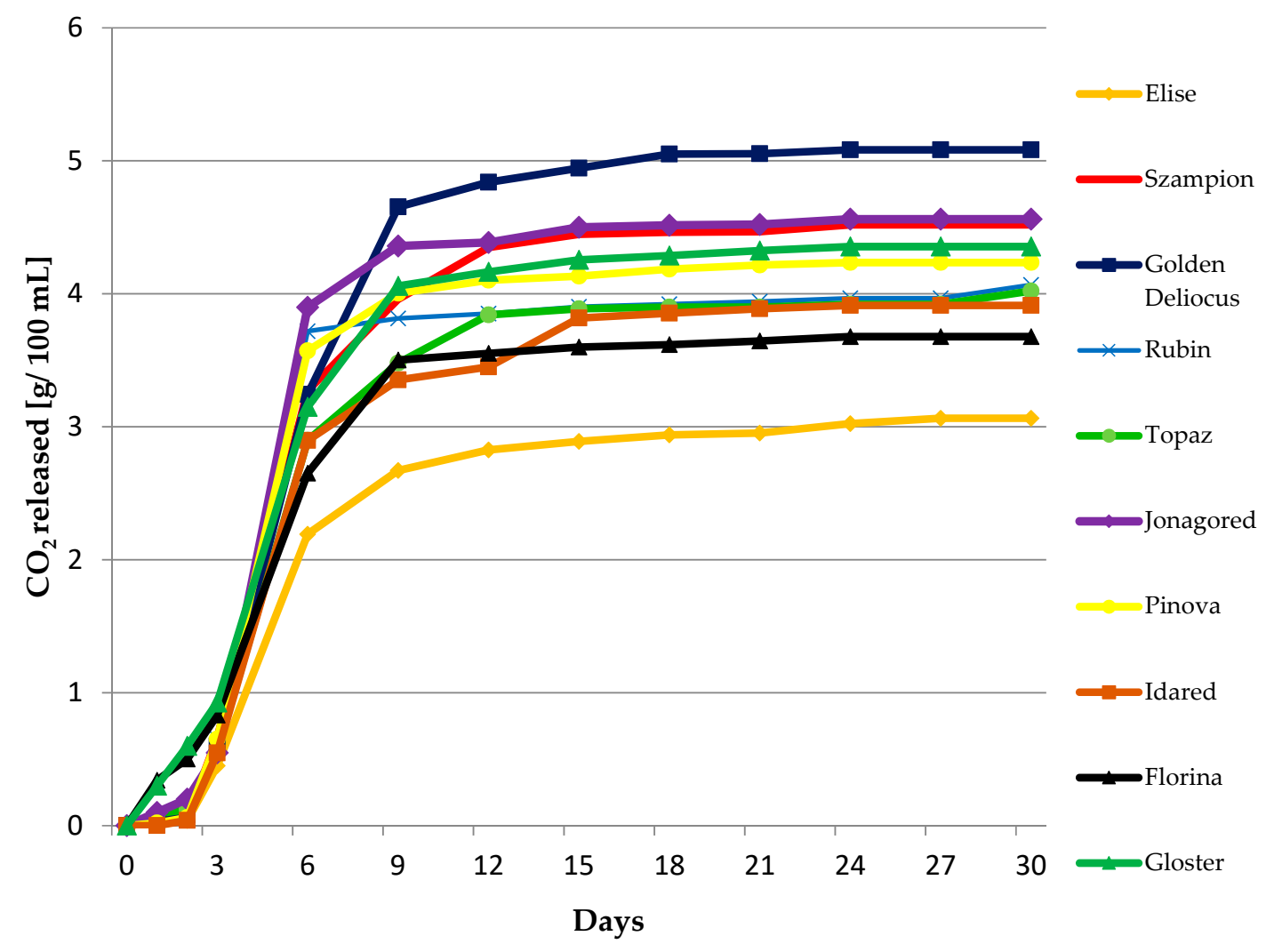

Figure 1. Fermentation dynamics of apple musts, $n=3$, STD $<5 \%$.

The fermentation efficiency ranged from $61.3 \%$ (Idared) to $94.7 \%$ (Gloster). In all samples, over $80 \%$ of reducing sugars was used during fermentation, with glucose being utilized in largest quantities (residual glucose from 0.03 to $0.28 \mathrm{~g} / \mathrm{L}$ ). During fermentation $S$. cerevisiae yeast initially uses glucose available in the medium, followed by other simple sugars and disaccharides [17]. The quantity of ethyl alcohol produced during the fermentation is mainly determined by the level of fermentable sugars. In apple musts not supplemented with sucrose, generally around $5 \%$ of ethanol is produced [10]. Ethanol content in fermented samples varied from 4.1 (Idared) to $6.3 \%$ vol. ethanol (Topaz) (Table 2). Similar or higher concentrations of ethanol in apple wines obtained from Rubin, Elise and Topaz fermented using Ethanol Red yeast were demonstrated by Tarko et al. (2018) [17]. 
Table 2. Selected chemical parameters of fresh and fermented musts obtained from various apple cultivars.

\begin{tabular}{|c|c|c|c|c|c|c|c|c|c|c|}
\hline \multirow{3}{*}{$\begin{array}{l}\text { Apple } \\
\text { Cultivars }\end{array}$} & \multicolumn{4}{|c|}{ Before Fermentation Processes } & \multicolumn{6}{|c|}{ After Fermentation Processes } \\
\hline & Total Extract & $\begin{array}{l}\text { Sugar-Free } \\
\text { Extract }\end{array}$ & $\begin{array}{l}\text { Titratable } \\
\text { Acidity }\end{array}$ & $\begin{array}{l}\text { Free Ammonia } \\
\text { Nitrogen (Fan) }\end{array}$ & Total Extract & $\begin{array}{l}\text { Sugar-Free } \\
\text { Extract }\end{array}$ & $\begin{array}{l}\text { Titratable } \\
\text { Acidity }\end{array}$ & $\begin{array}{l}\text { Free Ammonia } \\
\text { Nitrogen (fan) }\end{array}$ & $\begin{array}{l}\text { Ethanol } \\
\text { Content }\end{array}$ & $\begin{array}{c}\text { Fermentation } \\
\text { Efficiency }\end{array}$ \\
\hline & \multicolumn{3}{|c|}{$(g / L)$} & $(\mathrm{mg} / \mathrm{L})$ & \multicolumn{3}{|c|}{$(g / L)$} & $(\mathrm{mg} / \mathrm{L})$ & (\% vol.) & $(\%)$ \\
\hline Elise & $115.0 \mathrm{a} \pm 2.0$ & $24.2 b \pm 1.4$ & $4.86 \mathrm{~b} \pm 0.08$ & $53.1 \mathrm{~b} \pm 1.0$ & $9.0 \mathrm{e} \pm 1.0$ & $8.1 \mathrm{~d} \pm 0.8$ & $4.01 \mathrm{~cd} \pm 0.33$ & $24.7 \mathrm{ab} \pm 1.7$ & $5.1 b c \pm 0.2$ & $93.3 b \pm 0.6$ \\
\hline Rubin & $115.0 \mathrm{a} \pm 2.0$ & $15.0 \mathrm{c} \pm 0.8$ & $4.22 \mathrm{c} \pm 0.18$ & $43.3 c \pm 0.9$ & 13.0de \pm 1.5 & $12.3 b \pm 1.5$ & $5.02 b c \pm 0.67$ & $26.4 \mathrm{a} \pm 8.5$ & $6.2 \mathrm{a} \pm 0.2$ & $91.7 \mathrm{c} \pm 0.6$ \\
\hline Topaz & $120.0 \mathrm{a} \pm 1.0$ & $14.3 \mathrm{~d} \pm 0.3$ & $5.51 \mathrm{a} \pm 0.36$ & $31.9 \mathrm{e} \pm 0.1$ & $21.0 \mathrm{ab} \pm 1.0$ & $20.5 a \pm 1.3$ & $7.32 \mathrm{a} \pm 0.18$ & $13.2 \mathrm{bcd} \pm 1.9$ & $6.3 a \pm 0.3$ & $88.2 d \pm 0.5$ \\
\hline Szampion & $112.0 \mathrm{a} \pm 3.0$ & $14.3 \mathrm{~d} \pm 0.5$ & 3.39def \pm 0.13 & $45.4 c \pm 0.7$ & $14.0 \mathrm{~cd} \pm 2.0$ & $12.5 b \pm 0.7$ & $3.73 d \pm 0.47$ & $16.2 \mathrm{abc} \pm 1.8$ & $6.2 \mathrm{a} \pm 0.1$ & $93.8 \mathrm{~b} \pm 0.4$ \\
\hline $\begin{array}{l}\text { Golden } \\
\text { delicious }\end{array}$ & $119.0 \mathrm{a} \pm 1.0$ & $29.7 \mathrm{a} \pm 2.1$ & $3.28 \mathrm{ef} \pm 0.12$ & $16.6 \mathrm{f} \pm 1.7$ & $21.0 \mathrm{ab} \pm 1.5$ & $19.9 \mathrm{a} \pm 1.2$ & $4.91 \mathrm{~cd} \pm 0.44$ & $10.9 \mathrm{cde} \pm 2.9$ & $4.2 \mathrm{~d} \pm 0.3$ & $69.7 f \pm 0.3$ \\
\hline Jonagored & $104.0 \mathrm{~b} \pm 1.5$ & 13.0de \pm 1.3 & $3.32 \mathrm{def} \pm 0.19$ & $61.7 \mathrm{a} \pm 0.7$ & $19.0 \mathrm{bc} \pm 2.0$ & $18.4 \mathrm{a} \pm 1.7$ & $5.01 \mathrm{ab} \pm 0.68$ & $16.3 \mathrm{abc} \pm 3.56$ & $5.7 \mathrm{~b} \pm 0.2$ & $92.7 b c \pm 0.5$ \\
\hline Pinova & $114.0 \mathrm{a} \pm 2.0$ & $16.9 \mathrm{c} \pm 1.9$ & $2.94 f \pm 0.05$ & $28.1 \mathrm{e} \pm 0.6$ & 11.0de \pm 2.0 & $10.3 c \pm 0.7$ & $5.32 b c \pm 0.74$ & $8.3 \mathrm{de} \pm 5.96$ & $4.9 \mathrm{c} \pm 0.2$ & $74.7 \mathrm{e} \pm 0.3$ \\
\hline Idared & $104.0 \mathrm{~b} \pm 1.5$ & $4.9 \mathrm{f} \pm 0.1$ & $3.73 c d \pm 0.05$ & $13.7 f \pm 0.6$ & $15.0 \mathrm{~cd} \pm 1.0$ & $14.8 \mathrm{~b} \pm 1.8$ & $5.93 a b \pm 0.68$ & $13.3 b c d \pm 0.6$ & $4.1 b c \pm 0.5$ & $61.3 \mathrm{~g} \pm 0.4$ \\
\hline Florina & $104.0 \mathrm{~b} \pm 2.0$ & $20.3 b \pm 2.5$ & $2.11 \mathrm{~g} \pm 0.17$ & $29.3 \mathrm{e} \pm 1.5$ & $6.0 \mathrm{f} \pm 2.5$ & $5.2 \mathrm{e} \pm 0.7$ & $4.72 b c \pm 0.77$ & $22.5 \mathrm{abc} \pm 2.7$ & $5.2 b c \pm 0.2$ & $91.8 \mathrm{c} \pm 0.7$ \\
\hline Gloster & $104.0 \mathrm{~b} \pm 1.5$ & $21.0 \mathrm{~b} \pm 0.4$ & $3.69 \mathrm{cde} \pm 0.06$ & $41.8 \mathrm{~d} \pm 2.7$ & $18.0 \mathrm{ab} \pm 1.5$ & $17.7 \mathrm{a} \pm 1.3$ & $5.64 b c \pm 0.72$ & $18.2 \mathrm{abc} \pm 6.1$ & $5.3 c \pm 0.3$ & $94.7 \mathrm{a} \pm 0.4$ \\
\hline Significance & $* * *$ & $* * *$ & $* * *$ & $* * *$ & $* * *$ & $* * *$ & $* * *$ & $* * *$ & $* * *$ & $* * *$ \\
\hline
\end{tabular}

Same letters next to mean values within columns indicate the lack of statistically significant differences at $p<0.05 ; n=3 ; n s-$ not significant; $0.001=* * *$. 


\subsection{Volatile Compounds}

Qualitative and quantitative profile of terpenes in apple brandies has not been studied in detail so far. The presence of eugenol, chavicol and isoeugenol in apple fermented beverages was already shown [21]. In case of analyzed samples, eugenol was present in high concentrations (2.33-8.28 mg/L $100^{\circ}$ ). Significantly lower content of this compound (below $1 \mathrm{mg} / \mathrm{L}$ ) was detected in apple spirits analyzed by Rodríguez-Madrera and Mangas Alonso (2010) [22] and in plum brandies analyzed by Satora et al. (2016) [9] (below $0.2 \mathrm{mg} / \mathrm{L}$ ). Higher content of eugenol in apple spirits (6 mg/L) was demonstrated by Coldea et al. (2011) [6]. Analyzed brandies contained also high concentration of isoeugenol and $\beta$-ionone. Terpinen-4-ol was present in similar concentrations in all samples (about $1 \mathrm{mg} / \mathrm{L} 100^{\circ}$ ) (Table 3). This compound was characteristic for apple spirits analyzed by Bajer et al. (2017) [7]. The presence of $\beta$-citronellol distinguished four apple brandies and its highest content was noted in spirits obtained from fermented musts of Rubin and Szampion cultivars. Linalool oxide was present in all analyzed spirits $\left(0.51-0.77 \mathrm{mg} / \mathrm{L} 100^{\circ}\right)$ and its concentration was higher than in grape $\left(0.29 \mathrm{mg} / \mathrm{L} 100^{\circ}\right)$ and plum spirits $\left(0.21 \mathrm{mg} / \mathrm{L} 100^{\circ}\right)$ [8]. Myrcene and limonene were characteristic for apple brandies obtained from Topaz and Elise cultivars. Limonene is not commonly present in apple brandies. Its presence was also found in Earligold apple [23]. Gas chromatography- mass spectrometry method enables the detection of some other components, e.g., nerolidol, $\alpha$-phellandrene, o-cymene, $\alpha$-terpineol and $\beta$-damascenone (Table 4). Nerolidol with characteristic rose/keiskei/apple blossom flavor was present in highest concentration in all analyzed samples. This compound is also the major compound responsible for the aroma of grape spirits [24].

Ethyl acetate is the most abundant ester, generally exceeding $80 \%$ of all esters in fruit spirits [25]. In analyzed apple spirits, ethyl acetate was also predominant ester reaching over $25 \%$ of all volatile esters (Tables 3 and 4). The highest amounts of these compounds were detected in apple brandy obtained from Topaz cultivar $\left(199 \mathrm{mg} / \mathrm{L} 100^{\circ}\right)$ and the lowest from Jonagored apples $\left(105 \mathrm{mg} / \mathrm{L} 100^{\circ}\right)$. Much higher content of ethyl acetate (198.2-744.2 mg/L) in spirits obtained from distilled ciders was shown by Rodríguez-Madrera and Suárez Valles (2007) [26] which could be related to the involvement of particular microorganisms in fermentation. Acetate esters of higher alcohols and ethyl esters of fatty acids are significant volatile compounds in spirits. Peng et al. (2009) [27] reported that one of the key aroma components in ciders is isoamyl acetate. Samples analyzed in our research contained relatively high concentrations of that compound $\left(73-145 \mathrm{mg} / \mathrm{L} 100^{\circ}\right.$, Table 3). Analyzed apple brandies contained below $2 \mathrm{mg}$ of ethyl caproate per liter of $100^{\circ}$ (Table 3). Similar or higher concentrations were detected in apple spirits (1.92-12.82 mg/L) by Rodríguez-Madrera and Suárez Valles (2007) [26]. Ethyl caprate was detected almost at the same level in all apple spirits (about $8 \mathrm{mg} / \mathrm{L} 100^{\circ}$ ). The presence of this compound in apple brandies was confirmed by Bajer et al. (2017) [7]. Similarly, diethyl succinate was detected at similar levels in all analyzed samples. According to other researches, diethyl succinate commonly occurs in alcoholic beverages, e.g., plum brandies $(2 \mathrm{mg} / 100 \mathrm{~mL}$ $\left.100^{\circ}\right)$, mirabelle brandies $\left(3.5 \mathrm{mg} / 100 \mathrm{~mL} 100^{\circ}\right)$, Scotch whiskies $\left(0.3 \mathrm{mg} / 100 \mathrm{~mL} 100^{\circ}\right)$, cognac and Armagnac $\left(1 \mathrm{mg} / 100 \mathrm{~mL} 100^{\circ}\right)$ [28]. Ethyl laurate was present at relatively high concentrations in analyzed samples (about $4 \mathrm{mg} / \mathrm{L} 100^{\circ}$ ). The content of ethyl laurate was significantly lower (about $1 \mathrm{mg} / \mathrm{L} 100^{\circ}$ ) [29] in ten apple spirits purchased from local markets in Asturias (Spain) than its concentration demonstrated in the current study. This ester is characterized by fruity and waxy aroma and could be a characteristic component of apple spirits either because its concentration was rather high, and it was present in all samples. Methyl valerate was detected only in one sample (spirits obtained from Topaz cultivar). Ethyl caprylate has a fruity aroma and it is found in many species of fruits, e.g., apple, apricot, orange, grapefruit, guava, pineapple, passion fruit and mango [30]. This compound was present at highest concentrations in brandies obtained from Topaz, Szampion and Idared cultivars. Rodríguez-Madrera and Suárez Valles (2007) demonstrated lower content of this compound in apple spirits (3.03-15.36 mg/L) [26]. Gas chromatography-mass spectrometry method enables the detection of more than 30 other esters (Table 4). As in the case of terpenes, the spirits obtained from Gloster contained the smallest concentrations of esters. Some of esters were 
characteristic for spirits obtained from specific cultivar, for example benzyl acetate for Elise spirits and ethyl trans-4-decanoate for Jonagored. Benzoates (ethyl benzoate and benzyl benzoate) were a relatively small group of esters found in studied apple spirits present in low concentrations (Table 4). Benzoates in higher concentrations provide characteristic wintergreen-like flavor [31].

Table 3. Volatile compounds of brandies obtained by distillation of fermented musts from different apple cultivars by gas chromatography-flame ionization detector (GC-FID).

\begin{tabular}{|c|c|c|c|c|c|c|c|c|c|c|c|}
\hline \multirow[t]{2}{*}{ Terpenes } & Elise & Rubin & Topaz & Szampion & $\begin{array}{c}\text { Golden } \\
\text { Delicious }\end{array}$ & Jonagored & Pinova & Idared & Florina & Gloster & \multirow[t]{2}{*}{ Significance } \\
\hline & \multicolumn{10}{|c|}{$\left(\mathrm{mg} / \mathrm{L} 100^{\circ}\right)$} & \\
\hline Limonene & $0.39 a$ & $0.00 c$ & $0.21 b$ & $0.00 c$ & $0.00 c$ & $0.00 c$ & $0.00 c$ & $0.00 c$ & $0.00 c$ & $0.00 c$ & $* * *$ \\
\hline Linalool oxide & $0.51 b$ & $0.58 b$ & $0.75 a$ & $0.55 b$ & $0.53 b$ & $0.75 a$ & $0.65 \mathrm{ab}$ & $0.75 a$ & $0.77 \mathrm{a}$ & $0.76 \mathrm{a}$ & $* * *$ \\
\hline Linalool & $0.35 \mathrm{~b}$ & $0.35 b$ & $0.37 \mathrm{a}$ & $0.33 b$ & $0.35 b$ & $0.36 \mathrm{ab}$ & $0.37 a$ & $0.36 \mathrm{ab}$ & $0.35 \mathrm{ab}$ & $0.34 b$ & $* * *$ \\
\hline$(+)$-terpinen-4-ol & $0.94 a$ & $0.94 \mathrm{a}$ & $0.93 a$ & $0.92 a$ & $0.90 \mathrm{a}$ & $0.94 \mathrm{a}$ & $0.89 a$ & $0.90 \mathrm{a}$ & $0.92 \mathrm{a}$ & $0.88 \mathrm{a}$ & ns \\
\hline Citral & $0.28 \mathrm{a}$ & $0.26 a$ & $0.31 \mathrm{a}$ & $0.27 a$ & $0.29 a$ & $0.25 \mathrm{ab}$ & $0.30 \mathrm{a}$ & $0.28 \mathrm{a}$ & $0.24 \mathrm{ab}$ & $0.23 b$ & $* * *$ \\
\hline Geraniol & $0.18 \mathrm{a}$ & $0.19 a$ & $0.20 \mathrm{a}$ & $0.19 \mathrm{a}$ & $0.17 \mathrm{a}$ & $0.21 \mathrm{a}$ & $0.24 \mathrm{a}$ & $0.20 \mathrm{a}$ & $0.21 \mathrm{a}$ & $0.17 \mathrm{a}$ & ns \\
\hline$\beta$-ionone & $2.86 \mathrm{~d}$ & $3.41 \mathrm{c}$ & $6.41 \mathrm{a}$ & $5.01 \mathrm{~b}$ & $5.71 \mathrm{ab}$ & $3.96 \mathrm{c}$ & $6.48 \mathrm{a}$ & $5.90 \mathrm{ab}$ & $3.21 \mathrm{c}$ & $4.72 \mathrm{~b}$ & $* *$ \\
\hline Isoeugenol & $1.60 \mathrm{bc}$ & $1.88 \mathrm{ab}$ & $1.19 \mathrm{~g}$ & $1.67 \mathrm{~b}$ & $1.93 a$ & $1.42 \mathrm{~d}$ & $1.49 \mathrm{~cd}$ & $1.35 \mathrm{e}$ & $1.32 \mathrm{ef}$ & $1.27 \mathrm{f}$ & $* *$ \\
\hline Methyl eugenol & $0.18 \mathrm{~b}$ & $0.33 a$ & $0.17 \mathrm{~b}$ & $0.31 \mathrm{a}$ & $0.00 c$ & $0.33 a$ & $0.16 \mathrm{~b}$ & $0.00 c$ & $0.00 c$ & $0.28 \mathrm{a}$ & $* * *$ \\
\hline (-)-B-citronellol & $0.00 \mathrm{~d}$ & $0.38 \mathrm{ab}$ & $0.00 \mathrm{~d}$ & $0.37 \mathrm{ab}$ & $0.00 \mathrm{~d}$ & $0.19 b c$ & $0.00 \mathrm{~d}$ & $0.00 \mathrm{~d}$ & $0.15 c$ & $0.00 \mathrm{~d}$ & $* * *$ \\
\hline Eugenol & $6.46 \mathrm{~cd}$ & $7.55 c$ & $3.27 \mathrm{ef}$ & $8.28 \mathrm{a}$ & $7.58 \mathrm{ab}$ & $6.24 \mathrm{~cd}$ & $5.81 \mathrm{e}$ & $2.33 \mathrm{~g}$ & $5.57 \mathrm{e}$ & $2.97 \mathrm{f}$ & $* *$ \\
\hline Guaiacol & $0.26 \mathrm{~d}$ & $0.42 \mathrm{~cd}$ & $2.77 \mathrm{ab}$ & 2.31ab & $2.49 \mathrm{ab}$ & $2.81 \mathrm{a}$ & $2.02 \mathrm{aC}$ & $2.67 \mathrm{ab}$ & $2.56 \mathrm{ab}$ & $0.62 \mathrm{~cd}$ & $* * *$ \\
\hline Myrcene & $0.08 \mathrm{a}$ & $0.00 \mathrm{~b}$ & $0.11 \mathrm{a}$ & $0.00 \mathrm{~b}$ & $0.00 \mathrm{~b}$ & $0.00 \mathrm{~b}$ & $0.00 \mathrm{~b}$ & $0.00 \mathrm{~b}$ & $0.00 \mathrm{~b}$ & $0.00 \mathrm{~b}$ & $* * *$ \\
\hline \multicolumn{12}{|c|}{ Esters } \\
\hline Isoamyl acetate & $104 \mathrm{ab}$ & $86 b c$ & $145 \mathrm{a}$ & $84 \mathrm{bc}$ & $88 \mathrm{bc}$ & $79 b c$ & $73 b c$ & $96 a b c$ & $79 \mathrm{bc}$ & $90 \mathrm{bc}$ & ** \\
\hline Ethyl caproate & $1.84 \mathrm{a}$ & $1.89 \mathrm{a}$ & $1.84 \mathrm{a}$ & $1.87 \mathrm{a}$ & $1.82 \mathrm{a}$ & $1.88 \mathrm{a}$ & $1.78 \mathrm{a}$ & $1.84 \mathrm{a}$ & $1.85 \mathrm{a}$ & $1.84 \mathrm{a}$ & ns \\
\hline Ethyl caprate & $7.79 a$ & $7.87 \mathrm{a}$ & $8.15 a$ & $7.88 \mathrm{a}$ & $7.73 a$ & $7.94 a$ & $7.83 a$ & $7.79 a$ & $7.73 a$ & $7.66 a$ & ns \\
\hline Diethyl succinate & $5.64 \mathrm{~b}$ & $5.11 \mathrm{~cd}$ & $5.18 \mathrm{~cd}$ & $5.97 \mathrm{a}$ & $5.27 \mathrm{c}$ & $5.17 \mathrm{~cd}$ & $5.65 b$ & $5.69 b$ & $5.21 \mathrm{~cd}$ & $5.23 \mathrm{~cd}$ & $* * *$ \\
\hline Ethyl laurate & $4.50 \mathrm{bc}$ & $4.44 \mathrm{c}$ & $4.53 \mathrm{bc}$ & $4.47 \mathrm{c}$ & $4.58 \mathrm{bc}$ & $4.43 c$ & $4.49 \mathrm{c}$ & $4.81 \mathrm{a}$ & $4.80 \mathrm{a}$ & $4.44 \mathrm{c}$ & $* * *$ \\
\hline $\begin{array}{c}\text { Methyl } \\
\text { anthranilate }\end{array}$ & $70.6 a$ & $63.7 \mathrm{a}$ & $68.8 \mathrm{a}$ & $74.3 \mathrm{a}$ & $71.3 \mathrm{a}$ & $62.7 \mathrm{a}$ & $68.6 a$ & $69.0 \mathrm{a}$ & $61.4 \mathrm{a}$ & $69.3 \mathrm{a}$ & ns \\
\hline Methyl valerate & $0.00 \mathrm{~b}$ & $0.00 \mathrm{~b}$ & $19.9 \mathrm{a}$ & $0.00 \mathrm{~b}$ & $0.00 \mathrm{~b}$ & $0.00 \mathrm{~b}$ & $0.00 \mathrm{~b}$ & $0.00 \mathrm{~b}$ & $0.00 \mathrm{~b}$ & $0.00 \mathrm{~b}$ & $* * *$ \\
\hline Isopropyl acetate & $0.15 a$ & $0.08 \mathrm{~b}$ & $0.00 \mathrm{c}$ & $0.00 c$ & $0.00 \mathrm{c}$ & $0.00 c$ & $0.00 c$ & $0.00 \mathrm{c}$ & $0.00 \mathrm{c}$ & $0.00 \mathrm{c}$ & $* * *$ \\
\hline Ethyl caprylate & $18.7 \mathrm{~b}$ & $12.7 \mathrm{c}$ & $22.7 \mathrm{a}$ & $20.8 \mathrm{ab}$ & $9.7 \mathrm{~d}$ & $12.9 \mathrm{c}$ & $10.7 d$ & $22.7 \mathrm{a}$ & $12.8 \mathrm{c}$ & $8.8 \mathrm{~d}$ & $* * *$ \\
\hline $\begin{array}{l}\text { 2-phenylethyl } \\
\text { acetate }\end{array}$ & 112 de & $116 c$ & $117 \mathrm{c}$ & $118 \mathrm{bc}$ & $117 \mathrm{c}$ & $115 \mathrm{~cd}$ & $117 \mathrm{c}$ & $116 c$ & $112 \mathrm{~cd}$ & $120 \mathrm{a}$ & $* * *$ \\
\hline Ethyl acetate & $123 \mathrm{bc}$ & $192 b$ & $199 a$ & $192 b$ & $133 \mathrm{bc}$ & $105 c$ & $137 \mathrm{bc}$ & $127 \mathrm{bc}$ & $193 b$ & $191 b$ & $* * *$ \\
\hline \multicolumn{12}{|c|}{ Other Compounds } \\
\hline Acetaldehyde & $156 b$ & $199 \mathrm{ab}$ & $156 b$ & $92 c$ & $167 \mathrm{~b}$ & $174 \mathrm{~b}$ & $227 a$ & $178 \mathrm{~b}$ & $215 \mathrm{ab}$ & $204 a b$ & $* * *$ \\
\hline Methanol & $8462 b$ & $5424 d$ & $7684 c$ & $4588 \mathrm{e}$ & $9379 a$ & $8734 b$ & $9873 a$ & $8925 b$ & $8325 b c$ & $9236 a$ & $* * *$ \\
\hline Propanol & $162 a$ & $201 \mathrm{a}$ & $197 a b$ & $114 \mathrm{c}$ & $157 \mathrm{~b}$ & $199 \mathrm{ab}$ & $171 \mathrm{ab}$ & $113 c$ & $173 b$ & $111 \mathrm{c}$ & $* * *$ \\
\hline Butanol & $332 d$ & $244 \mathrm{e}$ & $690 \mathrm{a}$ & $620 \mathrm{~b}$ & $656 \mathrm{ab}$ & $321 \mathrm{~d}$ & $430 c$ & $696 a$ & $234 \mathrm{e}$ & $267 \mathrm{e}$ & $* * *$ \\
\hline Isobutanol & $10.2 \mathrm{c}$ & $19 b$ & $15.8 \mathrm{bc}$ & $12 \mathrm{c}$ & $8.1 \mathrm{c}$ & $19 \mathrm{~b}$ & $10 \mathrm{c}$ & $18.1 \mathrm{~b}$ & $34.0 \mathrm{a}$ & $26.3 \mathrm{ab}$ & $* * *$ \\
\hline Hexanol & $83.7 \mathrm{a}$ & $79.4 a$ & $78.3 \mathrm{a}$ & $81.5 \mathrm{a}$ & $82.1 \mathrm{a}$ & $79.4 a$ & $80.9 a$ & $83.7 \mathrm{a}$ & $91.5 \mathrm{a}$ & $81.5 \mathrm{a}$ & $* * *$ \\
\hline Amyl alcohols & $1576 b$ & 1151d & $1194 c$ & $1802 a$ & $1217 \mathrm{c}$ & $1153 \mathrm{~cd}$ & $1004 d$ & $867 \mathrm{e}$ & $1381 c$ & $983 e$ & $* * *$ \\
\hline
\end{tabular}

Color determination from lowest $(0 \%)$ to highest $(100 \%)$ concentration of volatile compounds.

Same letters next to mean values within rows indicate the lack of statistically significant differences at $p<0.05$; $n=3 ; n s-$ not significant, $0.001={ }^{* * *} ; 0.01=* *$.

Amyl alcohols are quantitatively the largest group of higher alcohols in analyzed distillates. They are responsible for the flavor of alcoholic beverages, and the quality of those drinks depends on their concentration [3]. The content of those compounds in analyzed samples ranged from $867 \mathrm{mg} / \mathrm{L}$ (Idared) to $1802 \mathrm{mg} / \mathrm{L} 100^{\circ}$ (Szampion). Spaho (2017) [25] confirmed that the largest share in the group of higher alcohols was assigned to amylic alcohol. The highest propanol concentration was noted in spirits obtained from Rubin cultivar $\left(201 \mathrm{mg} / \mathrm{L} 100^{\circ}\right)$. Rodríguez-Madrera and Suárez Valles (2007) [26] demonstrated that the concentration of that compound in spirits made from ciders ranged from 92.25 to $400.53 \mathrm{mg} / \mathrm{L} 100^{\circ}$. Butanol and isobutanol were present in all analyzed spirits. In the current study, concentration of isobutanol exceeded $230 \mathrm{mg} / \mathrm{L} 100^{\circ}$ and amounts of butanol ranged from 8.1 to $34.0 \mathrm{mg} / \mathrm{L} 100^{\circ}$. These values were lower than those reported by Coldea et al. (2011) [6] who analyzed apple brandies. In the case of hexanol, its concentration ranged from 78.3 to $91.5 \mathrm{mg} / \mathrm{L} 100^{\circ}$. Hexanol is responsible for the grassy scent in distillates, however, when the concentration of that compound exceeds $100 \mathrm{mg} / \mathrm{L} 100^{\circ}$, it deteriorates sensory properties of spirits [25]. 
The maximum acceptable methanol content in apple spirits distributed in the European Union is $12 \mathrm{~g} / \mathrm{L} 100^{\circ}$ vol. alcohol [32]. In the current study, the concentration of that substance in analyzed brandies was much lower (Table 3). In apple spirits tested by Croitoru et al. (2013) [33] methanol content reached $0.5 \%$ and the highest amount of this compound was characteristic to spirits obtained from apple-plum musts (1\%). Spirits obtained after cider distillation demonstrated significantly lower methanol content-from 203 to $679 \mathrm{mg} / \mathrm{L}$ [25]. Boiling temperature of methanol is only $64.7^{\circ} \mathrm{C}$ so most that compound is transferred to heads fraction.

The aroma of studied apple spirits was also evaluated according to other detected components such as aldehydes and ketones (Tables 3 and 4). Acetaldehyde, which dominates in aldehyde profile (about 90\%) in brandies [25], was present in all analyzed samples in concentration range from 92.4 to $226 \mathrm{mg} / \mathrm{L} 100^{\circ}$. Similar concentration of this compound was determined in different alcoholic beverages, e.g., wine distillates (37-111 mg/L 100), brandies (126-595 mg/L 100), Kirsch (110-170 mg/L 100), apple brandies $\left(140 \mathrm{mg} / \mathrm{L} 100^{\circ}\right)$ and plum brandies $\left(120 \mathrm{mg} / \mathrm{L} \mathrm{100}{ }^{\circ}\right)$ [28]. In low concentration aroma of acetaldehyde resembles cherry, hazelnuts and overripe apples, however, when the concentration of that compound exceeds $1.2 \mathrm{~g} / \mathrm{L} 100^{\circ}$, it deteriorates sensory properties of spirits. Other carbonyl compounds were present in relatively high concentrations and most of them were present in all analyzed samples (e.g., furfural, benzaldehyde and 6-methyl-5-hepten-2-one). Several substances from that group were characteristic in spirits from particular apple cultivars: 1,1-diethoxy-propane from Topaz and Idared cultivars; hexanal from Elise, Golden delicious and Topaz; and benzothiazole from Elise, Szampion, Topaz and Idared.

\subsection{Sensory Analysis}

All analyzed samples were described as clear and obtained maximal notes for that parameter. Among 10 analyzed apple spirits the highest scores for the parameter "overall note" obtained-Eliza, Rubin, Topaz and Florina. Brandies obtained from Topaz cultivar characterized sweet (5 points) and citrus (4 pts) aroma (Figure 2), which could be associated with the most diverse profile of volatile compounds, especially the highest concentration of most of terpenes, e.g., $\alpha$-phellandrene, $o$-cymene, $\alpha$-terpineol (SPME-GC-MS) and citral, myrcene (GC-FID). Only the aroma of spirits obtained from Florina cultivar was described as grassy ( 2 out of 5 pts) which could be related to the highest concentration of hexanol (grassy-green notes) and linalool oxide with characteristic tea tree aroma [4]. The aroma of spirits obtained from Golden delicious cultivar was described as floral (4 out of 5 pts) which could be related to the presence of isoeugenol (clove aroma), eugenol (clove aroma) and $\beta$-ionone with characteristic violet aroma [4]. Pungent aroma recognized in spirits obtained from Szampion cultivar could be attributed to the highest concentration of fusel alcohols and eugenol; the latter compound is responsible for clove aroma which could result in pungent and burning flavor [30]. Spirits made from Gloster cultivar which received lowest scores in sensory evaluation (overall note 3 out of 5 pts) did not contain any of analyzed terpenes, i.e., limonene, (-)- $\beta$-citronellol, myrcene and none of acetate esters analyzed with SPME-GC-MS. Pearson test indicated strong positive correlations between some descriptors (floral, sweet, fruity or citrus) and overall note. Moreover, there were negative correlations between pungent descriptor and overall note. 


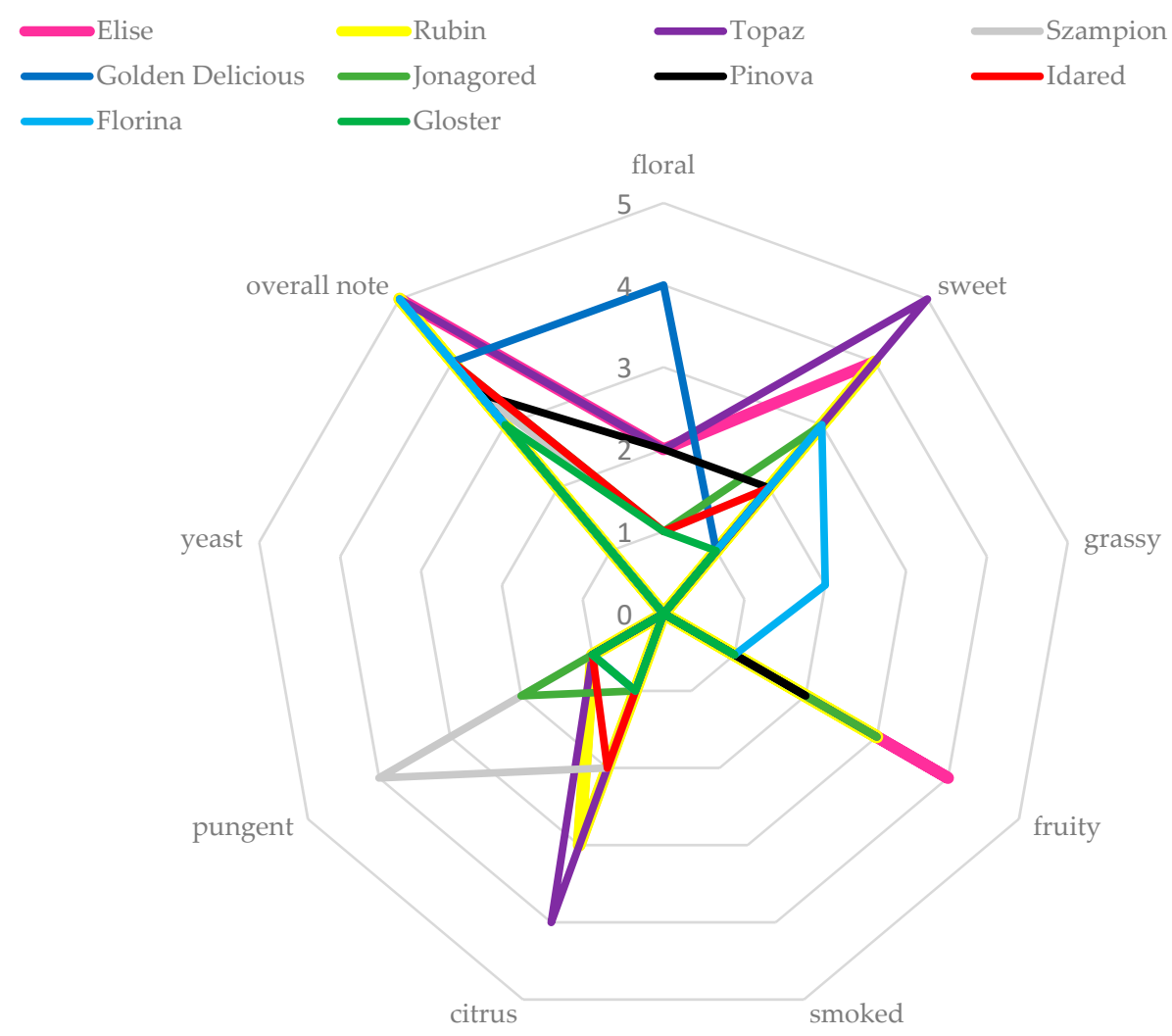

Figure 2. Characteristic aroma traits of apple spirits obtained from various apple cultivars fermented by Ethanol Red (Saccharomyces cerevisiae) yeast.

\section{Conclusions}

Our research proved that dessert apples could be used for the production of apple brandies and confirmed the hypothesis that the fruit cultivar significantly influences volatile profile and sensory characteristics of obtained spirit. Of the ten analyzed apple spirits, those obtained from Topaz, Rubin and Elise cultivars demonstrated the most diverse profile of volatile compounds. Moreover, their oenological parameters that are the most important in the production of alcoholic beverages were the most favorable. Brandies obtained from Gloster contained the smallest concentrations of esters and terpenes. Characteristic volatiles for brandies obtained from Topaz were limonene, myrcene, methyl valerate and 1,1-diethoxy-propane; Rubin — $\beta$-citronellol and isopropyl acetate; Elise-limonene, myrcene, benzyl acetate and isopropyl acetate; Szampion- $\beta$-citronellol; Idared-1,1-diethoxy-propane and Jonagored- ethyl trans-4-decanoate. Eugenol, $\beta$-ionone, $\beta$-damascenone and nerolidol were present at highest concentrations of terpenes in most of analyzed alcoholic beverages. Ethyl acetate was the most characteristic ester occurring in apple brandies and it dominated ester profile (about $30 \%$ of total esters). Results of the sensory analysis showed that the highest scores brandies were obtained from Topaz, Rubin, Elise and Florina. Those brandies demonstrated pleasant, sweet, fruity, citrus and alcoholic aroma. The results of this research will enrich the knowledge about the effect of the fruit cultivar on the quality of fruit brandies, and also enable producers to choose a cultivar of apples to produce beverages with new, unique characteristics. Topaz, Rubin and Elise cultivars were used for further unpublished studies on the production of apple brandies. 
Table 4. Aroma composition of apple spirits produced from different apple cultivars (solid-phase microextraction- gas chromatography-mass spectrometry $(\mathrm{SPME}-\mathrm{GC}-\mathrm{MS})\left(\mu \mathrm{g} / \mathrm{L} 100^{\circ}\right)$

\begin{tabular}{|c|c|c|c|c|c|c|c|c|c|c|c|c|}
\hline \multirow[t]{2}{*}{ Acetate Esters } & LRI $^{2}$ & Elise & Rubin & Topaz & Szampion & $\begin{array}{c}\text { Golden } \\
\text { Delicious }\end{array}$ & Jonagored & Pinova & Idared & Florina & Gloster & Sig. \\
\hline & \multicolumn{12}{|c|}{$\left(\mu \mathrm{g} / \mathrm{L} 100^{\circ}\right)$} \\
\hline Isobutyl acetate & 763 & $57.1 \mathrm{a}$ & $28.0 \mathrm{bc}$ & $18.8 \mathrm{~cd}$ & $45.6 \mathrm{ab}$ & $0.0 \mathrm{~d}$ & $10.1 \mathrm{~cd}$ & $4.5 \mathrm{~d}$ & $0.0 \mathrm{~d}$ & $0.0 \mathrm{~d}$ & $0.0 \mathrm{~d}$ & $* * *$ \\
\hline Butyl acetate & 799 & $36.9 b$ & $32.3 b$ & $6.5 b c$ & $90.6 \mathrm{a}$ & $0.0 \mathrm{c}$ & $35.0 \mathrm{~b}$ & $37.5 b$ & $27.6 \mathrm{bc}$ & $0.0 \mathrm{c}$ & $0.0 \mathrm{c}$ & $* * *$ \\
\hline 1-Butanol, 2-methyl-, acetate & 879 & $63.2 \mathrm{bc}$ & $125.4 b$ & $0.0 \mathrm{c}$ & $307.0 \mathrm{a}$ & $0.0 \mathrm{c}$ & $0.0 \mathrm{c}$ & $77.7 \mathrm{bc}$ & $63.5 \mathrm{bc}$ & $0.0 \mathrm{c}$ & $0.0 \mathrm{c}$ & $* * *$ \\
\hline Hexyl acetate & 1006 & $674.1 \mathrm{bd}$ & 2042.1a & $712.9 \mathrm{bc}$ & $906.4 b$ & $0.0 \mathrm{~d}$ & $89.4 \mathrm{~cd}$ & $377.8 \mathrm{bd}$ & $121.8 \mathrm{~cd}$ & $0.0 \mathrm{~d}$ & $0.0 \mathrm{~d}$ & $* * *$ \\
\hline Benzyl acetate & 1137 & $15.4 \mathrm{a}$ & $0.0 \mathrm{~b}$ & $0.0 \mathrm{~b}$ & $0.0 \mathrm{~b}$ & $0.0 \mathrm{~b}$ & $0.0 \mathrm{~b}$ & $0.0 \mathrm{~b}$ & $0.0 \mathrm{~b}$ & $0.0 \mathrm{~b}$ & $0.0 \mathrm{~b}$ & $* * *$ \\
\hline Octyl acetate & 1196 & $68.0 \mathrm{a}$ & $37.7 \mathrm{ab}$ & $49.3 \mathrm{ab}$ & $4.9 \mathrm{~b}$ & $0.0 \mathrm{~b}$ & $8.9 b$ & $5.8 \mathrm{~b}$ & $0.0 \mathrm{~b}$ & $0.0 \mathrm{~b}$ & $0.0 \mathrm{~b}$ & $* * *$ \\
\hline Decyl acetate & 1394 & $227.4 \mathrm{a}$ & $83.8 \mathrm{ab}$ & 107.5ab & $0.0 \mathrm{~b}$ & $0.0 \mathrm{~b}$ & $8.6 \mathrm{~b}$ & $0.0 \mathrm{~b}$ & $0.0 \mathrm{~b}$ & $0.0 \mathrm{~b}$ & $0.0 \mathrm{~b}$ & $* *$ \\
\hline \multicolumn{13}{|l|}{ Methyl and Ethyl Esters } \\
\hline Ethyl butyrate & 789 & $20.2 \mathrm{ab}$ & $55.9 \mathrm{a}$ & $55.5 a$ & $38.8 \mathrm{ab}$ & $0.0 \mathrm{~b}$ & $27.0 \mathrm{ab}$ & $4.6 \mathrm{~b}$ & $16.1 \mathrm{ab}$ & $0.0 \mathrm{~b}$ & $0.0 \mathrm{~b}$ & $* * *$ \\
\hline Ethyl-2-methylbutyrate & 841 & $0.0 \mathrm{c}$ & $0.0 \mathrm{c}$ & $2.7 \mathrm{~b}$ & $21.3 \mathrm{a}$ & $0.0 \mathrm{c}$ & $0.0 \mathrm{c}$ & $11.7 \mathrm{a}$ & $4.4 \mathrm{~b}$ & $0.0 \mathrm{c}$ & $0.0 \mathrm{c}$ & $* * *$ \\
\hline Ethyl 2-hydroxy-4-methylvalerate & 1060 & 20.0ad & $10.0 \mathrm{~cd}$ & $31.7 \mathrm{ab}$ & $34.5 \mathrm{a}$ & $0.0 \mathrm{~d}$ & 17.0ad & $6.7 \mathrm{~cd}$ & 17.2ad & $11.0 \mathrm{bd}$ & $23.3 \mathrm{ac}$ & $* *$ \\
\hline Methyl octanoate & 1108 & $14.3 \mathrm{a}$ & $36.6 a$ & $5.5 \mathrm{a}$ & $20.5 a$ & $0.0 \mathrm{a}$ & $9.4 \mathrm{a}$ & $20.6 a$ & $2.3 \mathrm{a}$ & $0.0 \mathrm{a}$ & $0.0 \mathrm{a}$ & ns \\
\hline Ethyl phenyl acetate & 1210 & $116.9 \mathrm{a}$ & $226.4 a$ & $110.5 \mathrm{a}$ & $787.3 \mathrm{a}$ & $47.3 \mathrm{a}$ & $282.6 a$ & $47.2 \mathrm{a}$ & 106.1a & $77.1 \mathrm{a}$ & $306.5 a$ & ns \\
\hline Ethyl 4-methyloctanoate & 1252 & $227.7 \mathrm{a}$ & $27.0 \mathrm{~b}$ & $23.5 b$ & $516.3 a$ & $24.1 \mathrm{~b}$ & $0.0 \mathrm{~b}$ & $15.5 b$ & $2.3 b$ & $0.0 \mathrm{~b}$ & $0.0 \mathrm{~b}$ & $* * *$ \\
\hline Ethyl trans-4-decanoate & 1357 & $0.0 \mathrm{~b}$ & $0.0 \mathrm{~b}$ & $0.0 \mathrm{~b}$ & $0.0 \mathrm{~b}$ & $0.0 \mathrm{~b}$ & $15.5 \mathrm{a}$ & $0.0 \mathrm{~b}$ & $0.0 \mathrm{~b}$ & $0.0 \mathrm{~b}$ & $0.0 \mathrm{~b}$ & * \\
\hline Ethyl 9-decenoate & 1366 & $55.6 a$ & $78.8 \mathrm{a}$ & $0.0 \mathrm{a}$ & $73.5 \mathrm{a}$ & $0.0 \mathrm{a}$ & $7.1 \mathrm{a}$ & $0.0 \mathrm{a}$ & $69.6 a$ & $5.3 a$ & $60.4 \mathrm{a}$ & ns \\
\hline Methyl dodecanoate & 1507 & 291.0ac & $632.7 \mathrm{ab}$ & $99.2 \mathrm{bc}$ & $681.1 \mathrm{a}$ & $1.2 \mathrm{c}$ & 217.2ac & $36.6 \mathrm{c}$ & $3.7 \mathrm{c}$ & $36.6 \mathrm{c}$ & $67.1 \mathrm{c}$ & $* * *$ \\
\hline Ethyl tetradecanoate & 1790 & $1470 \mathrm{~b}$ & $4584 \mathrm{ab}$ & 20,771a & $11,734 \mathrm{ab}$ & $16 b$ & $3246 a b$ & $111 b$ & $68 \mathrm{~b}$ & $360 \mathrm{~b}$ & $996 b$ & $* *$ \\
\hline Ethyl pentadecanoate & 1880 & $34.8 b$ & $70.0 \mathrm{~b}$ & $346.3 a$ & $125.3 \mathrm{ab}$ & $0.0 \mathrm{~b}$ & $84.5 b$ & $6.3 b$ & $0.0 \mathrm{~b}$ & $22.9 \mathrm{~b}$ & $34.9 \mathrm{~b}$ & $* *$ \\
\hline Methyl hexadecanoate & 1927 & $45.2 \mathrm{a}$ & $257.7 \mathrm{a}$ & $223.3 a$ & $390.1 \mathrm{a}$ & $0.0 \mathrm{a}$ & $208.7 a$ & $48.2 \mathrm{a}$ & $28.6 \mathrm{a}$ & $128.1 \mathrm{a}$ & $66.0 \mathrm{a}$ & $*$ \\
\hline Ethyl 9-hexadecenoate & 1977 & $102.3 b$ & $282.0 \mathrm{~b}$ & 1121.1a & $591.0 \mathrm{ab}$ & $0.0 \mathrm{~b}$ & $232.9 b$ & $30.5 b$ & $17.4 b$ & $82.5 b$ & $123.6 b$ & $* *$ \\
\hline Ethyl hexadecanoate & 1990 & $1878.5 b$ & $6249.2 b$ & $32,808.7 a$ & $16,294.2 \mathrm{ab}$ & $95.1 b$ & $6127.5 b$ & $383.6 \mathrm{~b}$ & $248.7 b$ & $2018.8 b$ & $3939.4 b$ & $*$ \\
\hline Methyl 9,12-octadecadienoate & 2147 & $227.8 b$ & $666.3 \mathrm{ab}$ & $322.8 \mathrm{~b}$ & 2088.1a & $0.0 \mathrm{~b}$ & $873.8 \mathrm{ab}$ & $58.0 \mathrm{~b}$ & $40.0 \mathrm{~b}$ & $438.2 \mathrm{ab}$ & $468.8 \mathrm{ab}$ & $*$ \\
\hline Ethyl octadecanoate & 2189 & $7.5 \mathrm{a}$ & $7.1 \mathrm{a}$ & $54.0 \mathrm{a}$ & $70.9 a$ & $18.5 \mathrm{a}$ & $21.5 \mathrm{a}$ & $0.0 \mathrm{a}$ & $3.1 \mathrm{a}$ & $10.9 \mathrm{a}$ & $29.8 \mathrm{a}$ & $*$ \\
\hline \multicolumn{13}{|l|}{ Benzoates } \\
\hline Ethyl benzoate & 1142 & $21.7 \mathrm{a}$ & $24.3 \mathrm{a}$ & $7.5 a$ & $23.54 a$ & $0.0 \mathrm{a}$ & $0.0 \mathrm{a}$ & $8.8 \mathrm{a}$ & $34.1 \mathrm{a}$ & $11.2 \mathrm{a}$ & $36.3 a$ & ns \\
\hline Benzyl Benzoate & 1750 & $49.7 \mathrm{ab}$ & $62.5 a$ & $40.2 \mathrm{abc}$ & $41.5 \mathrm{abc}$ & $0.0 \mathrm{c}$ & $39.9 a b c$ & $9.1 b c$ & $15.9 \mathrm{bc}$ & $46.5 \mathrm{ab}$ & $26.9 \mathrm{abc}$ & $* *$ \\
\hline
\end{tabular}


Table 4. Cont.

\begin{tabular}{|c|c|c|c|c|c|c|c|c|c|c|c|c|}
\hline \multirow[t]{2}{*}{ Acetate Esters } & LRI $^{2}$ & Elise & Rubin & Topaz & Szampion & $\begin{array}{c}\text { Golden } \\
\text { Delicious }\end{array}$ & Jonagored & Pinova & Idared & Florina & Gloster & Sig. \\
\hline & \multicolumn{12}{|c|}{$\left(\mu \mathrm{g} / \mathrm{L} 100^{\circ}\right)$} \\
\hline \multicolumn{13}{|l|}{ Other Esters } \\
\hline Hexyl butyrate & 1174 & $2.8 \mathrm{~b}$ & $28.1 \mathrm{a}$ & $6.7 \mathrm{ab}$ & $0.0 \mathrm{~b}$ & $0.0 \mathrm{~b}$ & $7.7 \mathrm{ab}$ & $3.6 \mathrm{~b}$ & $0.0 \mathrm{~b}$ & $0.0 \mathrm{~b}$ & $0.0 \mathrm{~b}$ & * \\
\hline Hexyl 2-methylbutanoate & 1222 & $98.3 \mathrm{~b}$ & $57.8 \mathrm{c}$ & $37.1 \mathrm{c}$ & $363.6 a$ & $0.0 \mathrm{~d}$ & $235.0 \mathrm{a}$ & $139.5 \mathrm{a}$ & $20.0 \mathrm{c}$ & $47.3 \mathrm{c}$ & $0.0 \mathrm{~d}$ & $* * *$ \\
\hline Isopentyl hexanoate & 1238 & $44.6 \mathrm{~b}$ & $21.7 \mathrm{~b}$ & $49.0 \mathrm{~b}$ & $193.3 \mathrm{a}$ & $0.0 \mathrm{c}$ & $21.7 \mathrm{~b}$ & $6.8 \mathrm{~b}$ & $4.9 \mathrm{~b}$ & $3.1 \mathrm{~b}$ & $6.2 \mathrm{~b}$ & $* * *$ \\
\hline Isobutyl octanoate & 1341 & $41.8 \mathrm{ab}$ & $17.0 \mathrm{ab}$ & $30.3 \mathrm{ab}$ & $65.2 \mathrm{a}$ & $0.0 \mathrm{~b}$ & $22.6 \mathrm{ab}$ & $9.5 \mathrm{ab}$ & $0.0 \mathrm{~b}$ & $10.2 \mathrm{ab}$ & $17.0 \mathrm{ab}$ & $*$ \\
\hline Hexyl hexanoate & 1372 & $36.3 \mathrm{a}$ & $63.9 \mathrm{a}$ & $160.7 \mathrm{a}$ & $40.9 a$ & $0.0 \mathrm{a}$ & $5.7 \mathrm{a}$ & $4.2 \mathrm{a}$ & $0.0 \mathrm{a}$ & $0.0 \mathrm{a}$ & $0.0 \mathrm{a}$ & ns \\
\hline á-Phenylethyl butanoate & 1411 & $26.4 \mathrm{ab}$ & $41.9 \mathrm{ab}$ & $63.3 \mathrm{a}$ & $36.5 a b$ & $0.0 \mathrm{~b}$ & 27.1ab & $12.8 \mathrm{~b}$ & $20.5 \mathrm{ab}$ & $4.7 \mathrm{~b}$ & $14.0 \mathrm{~b}$ & ** \\
\hline 3-methylbutyl octanoate & 1445 & $1350.0 \mathrm{a}$ & $1098.5 \mathrm{ab}$ & $904.8 \mathrm{abc}$ & $887.5 \mathrm{abc}$ & $0.0 \mathrm{c}$ & $438.2 \mathrm{abc}$ & $68.4 \mathrm{bc}$ & $33.5 b c$ & $60.5 b c$ & $216.1 b c$ & $* *$ \\
\hline 2-methylbutyl octanoate & 1449 & $176.9 \mathrm{a}$ & $123.6 \mathrm{ab}$ & $13.7 \mathrm{~b}$ & $73.4 \mathrm{ab}$ & $0.0 \mathrm{~b}$ & $53.3 \mathrm{ab}$ & $8.3 b$ & $0.0 \mathrm{~b}$ & $4.1 \mathrm{~b}$ & $22.7 \mathrm{~b}$ & $* *$ \\
\hline Propyl decanoate & 1472 & $67.2 \mathrm{a}$ & $26.6 b$ & $19.9 b$ & $21.1 \mathrm{~b}$ & $4.8 \mathrm{~b}$ & $17.7 \mathrm{~b}$ & $0.0 \mathrm{a}$ & $0.0 \mathrm{a}$ & $3.3 \mathrm{a}$ & $5.3 a$ & $* * *$ \\
\hline Dibutyl maleate & 1505 & $0.0 \mathrm{~b}$ & $0.0 \mathrm{~b}$ & $133.7 \mathrm{a}$ & $0.0 \mathrm{~b}$ & $0.0 \mathrm{~b}$ & $49.4 \mathrm{ab}$ & $16.9 \mathrm{~b}$ & $31.1 b$ & $28.6 b$ & $37.9 \mathrm{ab}$ & $* *$ \\
\hline 2-phenylethyl hexanoate & 1611 & $395.1 \mathrm{c}$ & $1508.3 \mathrm{ab}$ & $1040.9 \mathrm{~b}$ & $2827.8 \mathrm{a}$ & $30.8 \mathrm{c}$ & $1089.8 \mathrm{~b}$ & $605.0 \mathrm{bc}$ & $2917.8 \mathrm{a}$ & $195.2 \mathrm{c}$ & $448.6 c$ & $* *$ \\
\hline Isoamyl decanoate & 1641 & $3738 b$ & $8239 \mathrm{ab}$ & $50,019 a$ & $17,682 \mathrm{ab}$ & $0.0 \mathrm{~b}$ & $5378 a$ & $193 b$ & $119 \mathrm{~b}$ & $172 b$ & $839 \mathrm{~b}$ & * \\
\hline Isobutyl laureate & 1753 & $29.5 b$ & $47.3 b$ & $436.1 \mathrm{a}$ & $146.7 \mathrm{ab}$ & $0.0 \mathrm{~b}$ & $45.2 b$ & $0.0 \mathrm{~b}$ & $0.0 \mathrm{~b}$ & $4.0 \mathrm{~b}$ & $13.8 \mathrm{~b}$ & $* * *$ \\
\hline Hexyl decanoate & 1784 & $78.7 \mathrm{~b}$ & $172.1 \mathrm{ab}$ & $714.6 \mathrm{a}$ & $249.1 \mathrm{ab}$ & $42.7 \mathrm{~b}$ & $142.1 \mathrm{~b}$ & $86.1 \mathrm{~b}$ & $33.5 b$ & $45.4 \mathrm{~b}$ & $12.4 \mathrm{~b}$ & $* *$ \\
\hline 2-phenylethyl octanoate & 1820 & $487.0 \mathrm{~b}$ & $2092.5 b$ & $25,675.0 \mathrm{a}$ & $1892.1 \mathrm{~b}$ & $0.0 \mathrm{~b}$ & $2668.8 \mathrm{~b}$ & $459.6 b$ & $2087.6 b$ & $496.2 b$ & $1312.5 b$ & $* *$ \\
\hline Isoamyl laureate & 1844 & $56.5 \mathrm{ab}$ & $42.1 \mathrm{~b}$ & $50.0 \mathrm{~b}$ & $155.4 \mathrm{a}$ & $12.2 \mathrm{~b}$ & $46.7 \mathrm{~b}$ & $26.0 \mathrm{~b}$ & $30.5 b$ & $49.7 \mathrm{~b}$ & 107.9ab & $* * *$ \\
\hline \multicolumn{13}{|l|}{ Alcohols } \\
\hline 4-methyl-1-Pentanol & 821 & $25.9 \mathrm{de}$ & $121.1 \mathrm{ac}$ & $177.5 \mathrm{ab}$ & $179.5 \mathrm{a}$ & $0.0 \mathrm{c}$ & $79.7 \mathrm{~cd}$ & $104.7 \mathrm{bc}$ & $182.9 \mathrm{a}$ & 26.9de & $115.7 \mathrm{ac}$ & $* * *$ \\
\hline 3-methyl-1-Pentanol & 825 & $485.6 \mathrm{e}$ & 928.0ce & 1863.9ab & $1620.3 b c$ & $339.1 \mathrm{e}$ & 800.7de & $1460.2 \mathrm{bd}$ & 2520.1a & $407.8 \mathrm{e}$ & $629.6 \mathrm{e}$ & $* * *$ \\
\hline 3-Hexen-1-ol & 845 & $87.7 \mathrm{c}$ & $83.7 \mathrm{c}$ & $600.2 b$ & $369.7 \mathrm{bc}$ & $53.8 \mathrm{c}$ & $209.6 b c$ & $0.0 \mathrm{c}$ & $1710.1 \mathrm{a}$ & $0.0 \mathrm{c}$ & $1663.5 \mathrm{a}$ & $* * *$ \\
\hline 1-Heptanol & 954 & $75.4 \mathrm{c}$ & $368.0 \mathrm{~b}$ & $269.2 b c$ & $679.1 \mathrm{a}$ & $38.1 \mathrm{c}$ & $49.5 c$ & $141.8 \mathrm{bc}$ & $189.5 \mathrm{bc}$ & $29.6 c$ & $129.4 \mathrm{bc}$ & $* * *$ \\
\hline 1-Octanol & 1070 & $246.0 \mathrm{ac}$ & $518.9 a$ & $385.0 \mathrm{ac}$ & $479.5 \mathrm{ab}$ & $33.2 \mathrm{bc}$ & $47.8 \mathrm{bc}$ & $246.8 \mathrm{ac}$ & $101.6 \mathrm{ac}$ & $0.0 \mathrm{~b}$ & $27.3 \mathrm{bc}$ & ** \\
\hline Phenyl ethanol & 1084 & $10,865 b$ & $15,788 \mathrm{~b}$ & $47,775 \mathrm{ab}$ & $36,626 \mathrm{ab}$ & $86,592 a$ & $13,934 \mathrm{~b}$ & $43,232 \mathrm{ab}$ & $66,136 \mathrm{ab}$ & $15,692 b$ & $19,896 \mathrm{ab}$ & $* * *$ \\
\hline 1-Nonanol & 1156 & $120.6 b c$ & $1049.8 \mathrm{a}$ & $241.5 \mathrm{ac}$ & $1027.1 \mathrm{ab}$ & $89.7 \mathrm{c}$ & $0.0 \mathrm{c}$ & $138.0 \mathrm{bc}$ & $153.4 \mathrm{ac}$ & $119.5 b c$ & $122.7 \mathrm{bc}$ & $* *$ \\
\hline 1-Decanol & 1272 & $78.7 \mathrm{a}$ & $229.1 \mathrm{a}$ & $215.1 \mathrm{a}$ & $278.4 \mathrm{a}$ & $99.9 \mathrm{a}$ & $99.3 \mathrm{a}$ & $70.9 a$ & $89.8 \mathrm{a}$ & $52.9 \mathrm{a}$ & $136.5 \mathrm{a}$ & ns \\
\hline 6,10-dimethyl-5,9-Undecadien-2-ol & 1455 & $0.0 \mathrm{c}$ & $0.0 \mathrm{c}$ & $0.0 \mathrm{c}$ & $0.0 \mathrm{c}$ & $0.0 \mathrm{c}$ & $0.0 \mathrm{c}$ & $253.7 \mathrm{ab}$ & $9.8 \mathrm{c}$ & $335.9 a$ & $65.6 \mathrm{bc}$ & $* * *$ \\
\hline Phenol, 2,4-bis(1,1-dimethylethyl)- & 1490 & $563.1 \mathrm{~b}$ & $1039.6 a$ & $238.5 b$ & $1065.9 \mathrm{a}$ & $140.5 b$ & $766.9 \mathrm{~b}$ & $444.4 \mathrm{~b}$ & $456.4 \mathrm{~b}$ & $689.5 b$ & $478.5 b$ & $* * *$ \\
\hline 1-Tetradecanol & 1664 & $235.7 \mathrm{a}$ & $177.8 \mathrm{a}$ & $528.3 a$ & 261.7 & $45.8 \mathrm{a}$ & $241.5 \mathrm{a}$ & $25.1 \mathrm{a}$ & $27.4 \mathrm{a}$ & $135.7 \mathrm{a}$ & $152.2 \mathrm{a}$ & $* * *$ \\
\hline
\end{tabular}


Table 4. Cont.

\begin{tabular}{|c|c|c|c|c|c|c|c|c|c|c|c|c|}
\hline \multirow[t]{2}{*}{ Acetate Esters } & LRI $^{2}$ & Elise & Rubin & Topaz & Szampion & $\begin{array}{l}\text { Golden } \\
\text { Delicious }\end{array}$ & Jonagored & Pinova & Idared & Florina & Gloster & Sig. \\
\hline & \multicolumn{11}{|c|}{$\left(\mu \mathrm{g} / \mathrm{L} 100^{\circ}\right)$} & \\
\hline \multicolumn{13}{|l|}{ Aldehydes and Ketones } \\
\hline Hexanal & 777 & $165.6 \mathrm{~b}$ & $0.0 \mathrm{~b}$ & $698.9 a$ & $0.0 \mathrm{~b}$ & $132.5 \mathrm{~b}$ & $50.3 b$ & $0.0 \mathrm{~b}$ & $0.0 \mathrm{~b}$ & $0.0 \mathrm{~b}$ & $0.0 \mathrm{~b}$ & $* * *$ \\
\hline Furfural & 804 & $2305 \mathrm{~cd}$ & $2766 \mathrm{~cd}$ & $1975 \mathrm{~cd}$ & $1494 d$ & $1429 d$ & $9163 a$ & 6836ab & $4501 b c$ & $7586 a$ & $6702 \mathrm{ab}$ & $* * *$ \\
\hline Benzaldehyde & 925 & $1302 \mathrm{~cd}$ & $3374 a$ & $505 \mathrm{de}$ & $1907 \mathrm{bc}$ & $133 \mathrm{e}$ & 693de & $1690 \mathrm{bc}$ & $1270 \mathrm{~cd}$ & $2330 \mathrm{~b}$ & $1154 c d$ & $* * *$ \\
\hline 2-Methyltetrahydrothiophen-3-one & 952 & $938.3 a$ & $500.3 b c$ & $173.1 \mathrm{~d}$ & $540.6 \mathrm{bc}$ & $0.0 \mathrm{~d}$ & $395.9 c$ & $47.2 \mathrm{~d}$ & $89.3 \mathrm{~d}$ & $672.0 \mathrm{~b}$ & $629.3 b$ & $* * *$ \\
\hline 6-methyl-5-Hepten-2-one & 967 & 261.0de & 1280.1ad & $1691.5 \mathrm{ab}$ & 841.5be & $47.6 \mathrm{e}$ & 407.0ce & 1356.9ac & $2165.6 a$ & $16.2 \mathrm{e}$ & $113.8 \mathrm{e}$ & $* * *$ \\
\hline Nonanal & 1083 & $248.7 \mathrm{ab}$ & $238.2 \mathrm{ab}$ & $0.0 \mathrm{~b}$ & $617.8 \mathrm{a}$ & 213.3ab & 117.2ab & $178.3 \mathrm{ab}$ & $0.0 \mathrm{~b}$ & $87.5 \mathrm{ab}$ & $31.7 \mathrm{ab}$ & * \\
\hline Benzophenone & 1612 & 101.5ab & $17.9 \mathrm{~b}$ & 147.1a & $36.4 a b$ & $0.0 \mathrm{~b}$ & $44.5 \mathrm{ab}$ & $21.1 \mathrm{~b}$ & $14.2 \mathrm{~b}$ & $71.5 \mathrm{ab}$ & $0.0 \mathrm{~b}$ & $* *$ \\
\hline 3,7,11-trimethyl-2,6,10-Dodecatrienal & 1730 & $217.9 \mathrm{ab}$ & $433.6 \mathrm{a}$ & $96.1 \mathrm{ab}$ & $430.8 \mathrm{a}$ & $0.0 \mathrm{~b}$ & $149.2 \mathrm{ab}$ & $62.0 \mathrm{ab}$ & $56.8 \mathrm{ab}$ & $236.5 \mathrm{ab}$ & 201.1ab & * \\
\hline \multicolumn{13}{|l|}{ Terpenoids } \\
\hline$\alpha$-phellandrene & 1003 & $108.5 \mathrm{~b}$ & $112.6 \mathrm{~b}$ & $301.7 \mathrm{a}$ & $0.0 \mathrm{~d}$ & $0.0 \mathrm{~d}$ & $46.8 \mathrm{~cd}$ & $17.3 \mathrm{~cd}$ & $40.1 \mathrm{~cd}$ & $13.0 \mathrm{~cd}$ & $55.2 \mathrm{c}$ & $* * *$ \\
\hline o-cymene & 1014 & $122.0 \mathrm{ab}$ & $101.2 \mathrm{ab}$ & $158.2 \mathrm{a}$ & $0.0 \mathrm{~b}$ & $0.0 \mathrm{~b}$ & $31.1 \mathrm{ab}$ & $14.5 \mathrm{ab}$ & $19.0 \mathrm{ab}$ & $0.0 \mathrm{~b}$ & $27.1 \mathrm{ab}$ & $* *$ \\
\hline$\alpha$-terpineol & 1171 & $29.5 \mathrm{a}$ & $14.1 \mathrm{a}$ & $65.8 \mathrm{a}$ & 18.0a & $27.6 a$ & $42.4 a$ & $54.2 \mathrm{a}$ & $41.2 \mathrm{a}$ & $31.4 \mathrm{a}$ & $27.6 \mathrm{a}$ & $* * *$ \\
\hline$\beta$-damascenone & 1359 & $3608.2 a$ & $2273.0 \mathrm{ab}$ & $1579.5 \mathrm{ab}$ & 1905.1ab & $152.3 \mathrm{~b}$ & $1746.3 \mathrm{ab}$ & $1546.5 \mathrm{ab}$ & $1350.2 \mathrm{ab}$ & $346.5 b$ & $539.9 \mathrm{~b}$ & $* *$ \\
\hline$\beta$-famesene & 1458 & 913.4ab & $2682.7 \mathrm{a}$ & $367.1 b$ & $2770.5 \mathrm{a}$ & $18.3 b$ & $1121.4 \mathrm{ab}$ & $338.9 b$ & $259.2 b$ & $992.2 \mathrm{ab}$ & $824.3 \mathrm{ab}$ & $* * *$ \\
\hline a-farnesene & 1480 & $211.9 b$ & $643.6 \mathrm{a}$ & $36.9 c$ & $515.4 \mathrm{ab}$ & $0.0 \mathrm{c}$ & $401.0 \mathrm{~b}$ & $62.5 c$ & $30.0 c$ & $183.2 \mathrm{~b}$ & $138.1 \mathrm{~b}$ & $* * *$ \\
\hline Nerolidol & 1552 & $4062 \mathrm{ac}$ & $14,333 a$ & $2418 b c$ & $11,995 \mathrm{ab}$ & $15,000 \mathrm{c}$ & $2297 \mathrm{bc}$ & $2255 b c$ & $1218 b c$ & $3525 \mathrm{ac}$ & $3162 b c$ & $* *$ \\
\hline \multicolumn{13}{|l|}{ Other Compounds } \\
\hline 1,1-diethoxy-propane & 814 & $0.0 \mathrm{c}$ & $0.0 \mathrm{c}$ & $99.9 a$ & $0.0 \mathrm{c}$ & $0.0 \mathrm{c}$ & $0.0 \mathrm{c}$ & $0.0 \mathrm{c}$ & $43.4 \mathrm{~b}$ & $0.0 \mathrm{c}$ & $0.0 \mathrm{c}$ & $* * *$ \\
\hline Benzothiazole & 1186 & $266.0 \mathrm{a}$ & $0.0 \mathrm{a}$ & $275.7 \mathrm{a}$ & $324.2 \mathrm{a}$ & $0.0 \mathrm{a}$ & $0.0 \mathrm{a}$ & $0.0 \mathrm{a}$ & $362.5 a$ & $0.0 \mathrm{a}$ & $0.0 \mathrm{a}$ & $* *$ \\
\hline
\end{tabular}

Same letters next to mean values within rows indicate the lack of statistically significant differences at $p<0.05, n=3, n s-$ not significant; $0.001={ }^{* * *} ; 0.01={ }^{* *} ; 0.05={ }^{*} ;{ }^{2}$ LRI-linear retention index; the amount of compounds was determined semi-quantitatively by measuring the relative peak area of each identified compound, according to the National Institute of Standards and Technology (NIST) database, in relation to that of the internal standard. 
Supplementary Materials: The following are available online at http://www.mdpi.com/2218-273X/10/6/853/s1, Table S1: Details regarding HPLC method for each sugar and glycerol; Table S2: Pearson correlation coefficients between the intensities of the descriptors and the overall note of the brandies. Summary of statistical analyses carried out for results of sensory analysis.

Author Contributions: Conceptualization, M.J. and P.S.; methodology, M.J., P.S. and T.T.; software, M.J and P.S.; validation, M.J., P.S. and T.T.; formal analysis, M.J. and P.S.; investigation, M.J.; resources, M.J. and P.S.; data curation, M.J.; writing—original draft preparation, M.J. and P.S.; writing—review and editing, M.J., P.S. and T.T.; visualization, P.S.; supervision, P.S.; project administration, M.J.; funding acquisition, T.T. All authors have read and agreed to the published version of the manuscript.

Funding: This research was funded by the National Science Center, Poland (Project 2015/19/B/NZ9/01352).

Conflicts of Interest: The authors declare no conflicts of interest. The authors alone are responsible for the content and writing of the study.

\section{References}

1. Makosz, E. Przyszłość Polskich Jabłek. Biuletyn Informacyjny. Agencja Rynk. Rolnego 2015, 3, $10-16$.

2. Pischl, J. Destylaty Alkoholowe. Wytwarzanie, Teoria i Praktyka; Borus \& InterPolonus: Warszawa, Poland, $2010 ;$ p. 32.

3. Kostrz, M.; Satora, P. Związki lotne destylatów owocowych. Wybrane Zagadnienia Nauki o Żywności i Żywieniu. Pol. Tow. Technol. Żywności 2015, 1, 76-87. [CrossRef]

4. Kostrz, M.; Satora, P. Formation of terpenes in grapes and wines. Folia Pomer. Univ. Technol. Stetin. Agric. Aliment. Pisc. Zootech. 2018, 45, 31-38. [CrossRef]

5. Stanisz, M.; Sapnińska, E.; Pelech-Przybylska, K. Charakterystyka zanieczyszczeń chemicznych obniżających jakość spirytusu surowego. Zesz. Nauk. Politech. Łódzkiej Chem. Spożywcza I Biotechnol. 2009, 73, 105-121.

6. Coldea, T.E.; Socaciu, C.; Moldovan, Z.; Mudura, E. Minor volatile compounds in Traditional Homemade Fruit Brandies from Transylvania-Romania, as Determined by GC-MS Analysis. Not. Bot. Horti Agrobot. Cluj-Napoca 2011, 42, 530-537. [CrossRef]

7. Bajer, T.; Bajerová, P.; Surmová, S.; Kremr, D.; Ventura, K.; Eisner, A. Chemical profiling of volatile compounds of various home-made fruit spirits using headspace solid-phase microextraction. J. Inst. Brew. 2017, 123, 105-112. [CrossRef]

8. Dimitrov, D.; Ivanova, S. Aromatic profile of Bulgarian grape and fruit (plum) brandies. Ann. Food Sci. Technol. 2016, 17, 387-393.

9. Satora, P.; Kostrz, M.; Sroka, P.; Tarko, T. Chemical profile of spirits obtained by spontaneous fermentation of different varieties of plum fruits. Eur. Food Res. Technol. 2016, 243, 489-499. [CrossRef]

10. Maksimović, V.; Dragišić Maksimović, J. Science and Technology of Fruit Wine Production; Kosseva, M., Joshi, V.K., Panesar, P.S., Eds.; Academic Press: Cambridge, MA, USA, 2016; pp. 177-226.

11. International Organisation of Vine and Wine (OIV). Compendium of International Methods of Wine and Must Analysis; OIV: Paris, France, 2016; Volume 1.

12. Abernathy, D.G.; Spedding, G.; Starcher, B. Analysis of protein and total usable nitrogen in beer and wine using a microwell ninhydrin assay. J. Inst. Brew. 2009, 115, 122-127. [CrossRef]

13. Satora, P.; Tuszyński, T. Biodiversity of yeasts during plum Wegierka Zwykła spontaneous fermentation. Food Technol. Biotechnol. 2005, 43, 277-282.

14. Caldeira, I.; Belchior, A.P.; Clímaco, M.C.; Bruno de Sousa, R. Aroma profile of Portuguese brandies aged in chestnut and oak woods. Anal. Chim. Acta 2002, 458, 55-62. [CrossRef]

15. Lee, C. Common Nutrients and Nutraceutical Quality of Apples. N. Y. Fruit Q. 2012, 20, 3-8.

16. Joshi, V.K.; Attri, B.L. Science and Technology of Food Wine Production; Kosseva, M.R., Joshi, V.K., Panesar, P.S., Eds.; Academic Press: Cambridge, MA, USA, 2017; p. 299.

17. Tarko, T.; Kostrz, M.; Duda-Chodak, A.; Semik-Szczurak, D.; Sroka, P.; Senczyszyn, T. The effect of apple cultivars and yeast strains on selected quality parameters and antioxidant activity of fermented apple beverages. CyTA-J Food 2018, 16, 892-900. [CrossRef]

18. Dz, U. Dziennik Ustaw, 633, Rozporzadzenie Ministra Rolnictwa i Rozwoju Wsi w Sprawie Rodzajów Fermentowanych Napojów Winiarskich Oraz Szczegółowych Wymagań Organoleptycznych, Fizycznych, Chemicznych, Jakie Powinny Spetniać te Napoje; Government Legislation Center: Warszawa, Poland, 2003.

19. Alberti, A.; Giovanetti Vieira, R.; Françoise Drilleau, J.; Wosiacki, G.; Nogueira, A. Apple Wine Processing with Different Nitrogen Contents. Braz. Arch. Biol. Technol. 2011, 54, 551-558. [CrossRef] 
20. Satora, P.; Sroka, P.; Duda-Chodak, A.; Tarko, T.; Tuszyński, T. The profile of volatile compounds and polyphenols in wines produced from dessert varieties of apples. Food Chem. 2008, 111, 513-519. [CrossRef]

21. Xu, Y.; Fan, W.; Qian, M. Characterization of Aroma Compounds in Apple Cider Using Solvent-Assisted Flavor Evaporation and Headspace Solid-Phase Microextraction. J. Agric. Food Chem. 2007, 55, 3051-3057. [CrossRef]

22. Rodríguez-Madrera, R.; Mangas Alonso, J.J. Distribution of the principal minor volatiles during cider distillation in 'alquitara'. Acta Aliment. 2011, 40, 262-269. [CrossRef]

23. Rapparini, F.; Baraldi, R.; Facini, O. Seasonal variation of monoterpene emission from Malus domestica and Prunus avium. Phytochemistry 2001, 57, 681-687. [CrossRef]

24. Wei, X.F.; Ma, X.L.; Cao, J.H.; Sun, X.Y.; Fang, Y.L. Aroma characteristics and volatile compounds of distilled Crystal grape spirits of different alcohol concentrations: Wine sprits in the Shangri-La region of China. Food Sci. Technol. 2018, 38, 50-58. [CrossRef]

25. Spaho, N. Distillation-Innovative Applications and Modeling; Mendes, M., Ed.; InTech: London, UK, 2018; pp. 129-152.

26. Rodríguez-Madrera, R.; Suárez Valles, B. Determination of volatile compounds in cider spirits by gas chromatography with direct injection. J. Chromatogr. Sci. 2007, 45, 428-434. [CrossRef]

27. Peng, B.; Yue, T.; Yuan, Y. Analysis of key aroma components in cider from Shaanxi (China) Fuji apple. Int. J. Food Sci. Technol. 2009, 44, 610-615. [CrossRef]

28. Nykänen, L.; Suomalainen, H. Aroma of Beer, Wine and Distilled Alcoholic Beverages; Akademie-Verlag: Berlin, Germany, 1983.

29. Mangas, J.; Rodríguez, R.; Blanco, D. Solid-phase extraction and determination oftrace aroma and flavour components in cider by GC-MS. Chromatographia 1996, 42, 101-105. [CrossRef]

30. Burdock, G.A. Fenaroli's Handbook of Flavor Ingredients, 5th ed.; CRC Press: New York, NY, USA, 2006; Volume 1330, p. 616.

31. De Rovira, D. Flavor Ingredient and Miscellaneous. Dictionary of Flavors, 3rd ed.; John Wiley \& Sons Ltd.: New York, NY, USA, 2017; p. 33. [CrossRef]

32. OJ L 39, 13.2.2008. REGULATION (EC) No 110/2008 OF THE EUROPEAN PARLIAMENT AND OF THE COUNCIL of 15 January 2008 on the definition, description, presentation, labelling and the protection of geographical indications of spirit drinks and repealing Council Regulation (EEC) No 1576/89. Available online: http://data.europa.eu/eli/reg/2008/110(1)/oj (accessed on 22 April 2020).

33. Croitoru, M.D.; Topor, E.; Fogarasi, E. A survey on the metanol content of Home distilled alcoholic beverages in Transylvania (Romania). Acta Med. Marisiensis 2013, 59, 206-208. [CrossRef] 